\title{
Tbxl interacts genetically with Vegfr3 to regulate cardiac lymphangiogenesis in mice
}

Stefania Martucciello ${ }^{1,5 \#, \text { Maria Giuseppina Turturo }}{ }^{2 \#}$, Sara Cioffi ${ }^{2}$, Li Chen ${ }^{3}$, Antonio Baldini $^{2,4}$, Elizabeth Illingworth ${ }^{2,5 *}$

${ }^{1}$ IRCCS Neuromed, 86077, Pozzilli, Italy

${ }^{2}$ Institute of Genetics and Biophysics “ABT”, CNR, 80131, Naples, Italy

${ }^{3}$ University of Houston, Houston, Texas, USA

${ }^{4}$ University of Naples, Federico II, 80131, Naples, Italy

${ }^{5}$ University of Salerno, 84084, Fisciano, Italy

\# equal contributing authors

* Corresponding author

Prof. Elizabeth Illingworth, Ph.D

Department of Chemistry and Biology

University of Salerno

Via Giovanni Paolo II, 132

84084 Fisciano, Italy

email:eillingworth@unisa.it

Tel: +39 089/968121 


\begin{abstract}
The transcription factor $T B X 1$ is the major gene implicated in $22 \mathrm{q} 11.2$ deletion syndrome. The complex clinical phenotype includes vascular anomalies and a recent report presented new cases of primary lymphedema in 22q11.2DS patients. We have previously shown that Tbx1 activates Veg fr3 gene expression in lymphatic endothelial cells and that this activation is critical for lymphatic vessel development in prenatal mice and for their survival post-natally. Using loss-of-function genetics and transgenesis, we show a strong genetic interaction between Tbxl and Vegfr3 in cardiac lymphangiogenesis that causes cardiac lymphatic vessel anomalies in compound heterozygotes. Intriguingly, different aspects of the cardiac lymphatic phenotype were regulated independently by the two genes. $\mathrm{Tb} x 1^{\mathrm{Cre}}$-activated Vegfr3 transgene expression was able to rescue the morphological abnormalities in the cardiac lymphatic vessels of compound heterozygotes, but it did not rescue the severe cardiac lymphatic vessel hypoplasia observed in Tbxl homozygotes. Moreover, our study revealed a differential sensitivity between the ventral and dorsal cardiac lymphatic networks to the effects of altered Tbxl and Vegfr3 gene dosage. Overall, our study demonstrates that a fine dosage balance between Tbxl and Vegfr3 is required to regulate the number and morphology of cardiac lymphatic vessels.
\end{abstract}




\section{INTRODUCTION}

Tbxl is a major developmental gene whose critical role in lymphangiogenesis has been demonstrated in the mouse (1). In mice, in the absence of Tbxl, most lymphatic vessels are lost around embryonic day (E)16.5. We have shown that Tbx1 regulates Vegfr3 expression by binding to an intragenic enhancer element in the endogenous Vegfr3 gene. In Tbxl germline mutants and in ECspecific Tbx1 conditional mutants, Vegfr3 expression in lymphatic ECs is lost between E15.5 and 16.5, which led us to hypothesize that lymphatic vessels in Tbxl mutants are not maintained because of reduced Vegfr3 expression in lymphatic ECs (1).

In this study, we have investigated the interplay between Tbx1 and Vegfr3 in cardiac lymphangiogenesis by manipulating the dosage of both genes in ECs. We focused our attention on cardiac lymphangiogenesis because the cardiac lymphatic phenotype is severe in Tbxl mutants (1) and because cardiac neo-lymphangiogenesis has been implicated in the response to ischemia (2) (3) (4).

In the mouse, the cardiac lymphatic network develops from lymphatic ECs that migrate from extra-cardiac tissues into the heart around E12.5. As embryonic development progresses, an extensive network of sub-epicardial lymphatic vessels forms, extending over the heart from base to apex and covering mainly the left ventricle, both ventrally and dorsally, while fewer lymphatic vessels extend over the right ventricle (5)(2). The cardiac lymphatic vessel network is complete at postnatal day (P) 15.

As in other organs, in the heart, the primary role of the lymphatic vessels is to maintain fluid homeostasis and transport immune cells. In the adult heart, tissue fluid absorbed by the lymphatic capillaries drains into two main pre-collector vessels within the heart, that run along the left conal vein dorsally, and the left cardiac vein ventrally, and ultimately into the mediastinal lymph nodes. Studies in mice show that acute inflammation provokes neolymphangiogenesis, which is important for antigen clearance and for the resolution of inflammation (6)(7). Recently, Klotz et al., showed that cardiac injury (myocardial infarction) provokes a robust neolymphangiogenic response and upregulation of developmental lymphatic genes (Vegfr3, Prox1, Lyve1). Furthermore, post-infarct cardiac function improved after treatment with recombinant Vegf-C (2), suggesting that the Vegf-CVegfr3 axis is important for responding to pathological conditions of the heart.

In this study, we use genetic experiments to demonstrate a strong interaction between Tbx1 and Vegfr3 in cardiac lymphangiogenesis. Increasing endothelial expression of Vegfr 3 was sufficient to rescue partially the cardiac lymphatic phenotype in compound heterozygous mutants. However, it did not improve cardiac lymphatic development in Tbxl homozygous mutants, which lack lymphatic 
vessels in most tissues, including the heart. Overall, our data suggest that a dosage balance between Tbx1 and Vegfr3 is critical for cardiac lymphatic development.

\section{RESULTS}

\section{Tbx1 and Vegfr3 expression overlap in cardiac lymphatics}

We used a lacZ reporter allele (8) and monitored Tbxl expression by $\beta$-galactosidase ( $\beta$-gal) activity in $T b x l^{\text {lacz/+ }}$ hearts at E18.5 (Fig. 1). This revealed $\beta$-gal+ vessel-like structures on the surface of the heart that resembled lymphatic vessels (Fig. 1A, a, a'). To determine whether these were in fact lymphatic vessels, we immunostained the hearts of wild type littermates with anti-Vegfr3 antibody, which specifically labels lymphatic endothelial cells at this developmental stage (9). Results indicated that Tbxl (Fig. 1A, a, a') and Vegfr3 (Fig. 1A, b, b') had near identical expression, indicating that they are co-expressed in most cardiac lymphatic vessels at this stage of development. Note that Tbxl is also expressed in the conotruncus (arrow in Fig. 1A, a), as previously reported (10) (11).

In order to understand where Tbx1 and Vegfr3 may interact to promote cardiac lymphangiogenesis, we first mapped the distribution of Tbxl-expressing cells and their descendants in embryonic mouse hearts, and then immunostained the same hearts with anti-Vegfr3. For this, we crossed $T b x I^{\mathrm{Cre} /+}$ mice with R26R-lacZ reporter mice (12) and evaluated Tbxl-induced recombination in the hearts of $T b x 1^{C r e /+;} R 26 R$ embryos at E18.5 by $\beta$-gal staining. The hearts were then immunostained with anti-Vegfr3 antibody. Results revealed extensive overlap between Vegfr3 expression and $\beta$-gal staining in cardiac lymphatic vessels (Supplementary Fig. S1), indicating that Tbxl-expressing cells and their descendants populate the majority of cardiac lymphatic vessels. Furthermore, the lack of $\beta$-gal+; Vegfr3-negative vessels indicates that in the mouse heart, they contribute exclusively to lymphatic vessels and not to veins and arteries. Tbx1 ${ }^{\text {Cre }}$ activates recombination in cardiac muscle of the right ventricle but this tissue does not express Vegfr 3 , thereby excluding this tissue as a potential site of interaction. We also evaluated the distribution of Tbx1expressing cells and their descendants using an alternative reporter (Rosa $\left.{ }^{m T-m G}\right)$ (13). For this, we crossed $T b x 1^{C r e /+}$ mice with $\operatorname{Rosa}^{m T-m G}$ mice and analyzed the hearts of Tbx1 ${ }^{\text {Crel+; }} \operatorname{Rosa}^{m T-m G}$ embryos at E18.5, where Tbxl-expressing cells and their descendants were labelled by $T b x 1^{C r e}$-activated expression of a fluorescent (GFP) reporter (Fig. 1A, c, c'). Results were comparable to those obtained with the R26R-lacZ reporter.

In the mouse, the first cardiac lymphatic vessels form around E14.5 at the sinus venosus on the dorsal surface of the heart and at the base of the aorta on the ventral surface, from where they extend in a basal to apical direction (5)(2). We asked what is the earliest time point of Tbxl expression in 
cardiac lymphatic vessels? To address this question we performed $\beta$-gal staining and Lyve1 immunostaining on Tbxl ${ }^{\text {lacz/+ }}$ hearts at E14.5 and E15.5 (anti-Lyve1 labels lymphatic ECs). Results showed that at E14.5 (Fig. 1B, a-d) and E15.5 (Fig. 1B, e-h), on the dorsal surface of the heart, lymphatic vessels (Lyve1+) at the sinus venosus (Fig. 1B, c, c', g, g') also expressed Tbxl ( $\beta$-gal+) (arrows in d' and h'). This was less evident on the ventral surface of the E14.5 heart, because Tbxl is highly expressed in the conotruncus (arrow in b'), where the first ventral lymphatic vessels form (arrows in a'). However, at E15.5, a small network of Tbxl-expressing lymphatic vessels was visible on the ventral surface of the outflow tract (white arrow in $\mathrm{f}^{\prime}$ ) and heart (black arrow in $\mathrm{f}^{\prime}$ ). Thus, we conclude that $T b x l$ is expressed in the earliest forming cardiac lymphatic vessels.

\section{$T b x 1$ is expressed and required in the early phases of cardiac lymphatic development.}

In order to pinpoint the critical time requirement for Tbxl in cardiac lymphatic development, we crossed TgCAGG-CreER ${ }^{\mathrm{TM}} ; \mathrm{Tbx} 1^{\text {lacZ/+ }}$ mice with $T b x 1^{\text {floxfflox }}$ mice and inactivated $T b x 1$ in a timespecific manner, as previously described (10). We injected pregnant female mice with a single dose of tamoxifen (TM) at E10.5, E11.5, E12.5, E14.5 and E16.5. We then harvested embryos at E18.5 and analyzed the cardiac lymphatic vessels by Vegfr3 immunostaining (Fig. 1C). Results showed that after injection of TM at E10.5 (Fig. 1C, b) lymphatic vessels were present on the surface of the sinus venosus (arrows in b) but not in the ventricular myocardium. TM injection at E11.5 and E12.5 (Fig. 1C, c, d) resulted in cardiac lymphatic vessel hypoplasia, while after TM injection at E14.5 and E16.5, the cardiac lymphatic vessel network was indistinguishable from controls (Fig. 1C, a, e, f). These results indicate that the critical time requirement for Tbxl in cardiac lymphangiogenesis is before E14.5. Thus, Tbxl may be required for the migration of lymphatic ECs into the heart or for their coalescence to form cardiac lymphatic vessels.

\section{Tbx1 and Vegfr3 interact genetically in cardiac lymphatic development}

We next tested whether Tbxl and Vegfr3 interact genetically to regulate cardiac lymphangiogenesis. For this, we crossed $T b x 1^{\mathrm{Cre} /+}$ with $\operatorname{Veg} f r 3^{\text {flox/+ }}$ mice (14) and analyzed cardiac lymphatic vessels in E18.5 Tbx $\mathrm{Cre}^{\mathrm{Cr}+}$; Vegfr $3^{\text {flox/+ }}$ embryos by whole-mount immunostaining with antiLyve-1 antibody. We also generated $V e g f r 3^{+/-}$(germline heterozygous) mice by crossing Vegfr $3^{\text {flox/+ }}$ mice with ubiquitous Cre-expressing mice. We first analyzed the cardiac lymphatic vessels in Tbx $1^{\mathrm{Cre} /+}$ and $V e g f r 3^{+/}$embryos compared to wild type littermates. We quantified the number, length and width (ratio length/area) of lymphatic vessels in at least five E18.5 embryos per genotype, using the ImageJ software. In $\mathrm{Tb} x \mathrm{I}^{\mathrm{Cre} /+}$ embryos, we found no differences in the number, length or width 
of cardiac lymphatic vessels on either the ventral or dorsal surfaces of the heart (Fig. 2A, b, b' and Fig. 2C, a, a', b, b', c, c') compared to WT controls (Fig. 2A, a, a'). In $\operatorname{Veg} f r 3^{+/}$embryos, the number and length of cardiac lymphatic vessels was not different to WT littermates, but both ventral and dorsal lymphatic vessels were moderately dilated (Fig. 2B, b, b', Fig. 2D, c, c'). We next analyzed compound heterozygous embryos $\left(\mathrm{Tbx} \mathrm{I}^{\mathrm{Cre} /+} ; \mathrm{Vegfr} 3^{\text {flox/+ }}\right)$, and found that the cardiac lymphatic network was strongly reduced (Fig. 2A, c) compared to controls ( $T b x 1^{\mathrm{Cre} /+}$, Vegfr $3^{+/-}$, WT), especially on the ventral surface of the heart where, in most embryos, only a few lymphatic vessels were present around the base of the pulmonary trunk and subpulmonary myocardium (arrow in Fig. 2A, c). The dorsal lymphatic network presented as a reduced network of short, dilated vessels that failed to extend to the apex of the heart (arrow in Fig. 2A, c'). Quantitative analysis confirmed a reduction in the number (Fig. 2C, a, a'), length (Fig. 2C, b, b') and width (Fig. 2C, c, c') of ventral and dorsal cardiac lymphatic vessels in these mutants. Together, these results demonstrate the existence of a strong genetic interaction between Tbx1 and Vegfr3 in cardiac lymphangiogenesis that affects the number and morphology of the lymphatic vessels.

We also examined the intestinal lymphatic vessels of the same embryos by Lyve-1 immunostaining. Results revealed that also in this tissue, there was lymphatic vessel hypoplasia in Tbx $1^{\mathrm{Cre} /+}$; Vegfr $3^{\text {flox/+ }}$ embryos, in both the membranous mesentery and in the intestinal wall, compared to controls $\left(\operatorname{Tb} x 1^{\mathrm{Cre} /+}, \mathrm{Vegfr} 3^{+/}\right.$, WT), although the phenotype was more variable than in the heart (Supplementary Fig. S2). Thus, we conclude that the genetic interaction between Tbxl and Vegfr3 is not limited to cardiac lymphatic development.

\section{Generation of a Cre-inducible Vegfr3 transgenic mouse}

We have hypothesized that lymphatic vessel hypoplasia in Tbxl mutants is secondary to reduced Vegfr3 in Tbxl-depleted LECs (1) and here we provide evidence of a genetic interaction between the two genes. We next tested whether increasing Vegfr3 expression in Tbxl-expressing tissues, that includes lymphatic ECs, is sufficient to restore normal lymphatic development in single and compound Tbxl mutants. We generated a transgenic mouse (named TgVegfr3) that expresses Vegfr3 upon Cre-induced recombination. For this, we generated a Vegfr3-expressing transgene containing a full length murine $\operatorname{Vegfr} 3 \mathrm{cDNA}$ (Fig. 3A) that we electroporated into E14Tg2A.4 mouse embryonic stem cells (Bay Genomics, CA). We obtained and sequence-verified six clones that had a single integration site and a single copy of the transgene (Fig. 3B, C). Two of these clones were injected into mouse blastocysts. We obtained germline transmission of the transgene in four male offspring. We then characterized the transgenic Vegfr3 protein in cells and in mice. We first tested whether Cre activation of the transgene led to the production of a full length Vegfr3 protein. For this, 
we co-transfected $\mathrm{C} 2 \mathrm{C} 12$ cells, which do not express Vegfr3, with the transgene, and a Cre-expressing vector. Western blotting with anti-Vegfr3 antibody performed $24 \mathrm{~h}$ after transfection revealed the presence of a protein of the expected length of 195 Kda (Fig. 3D). Furthermore, anti-Vegfr3 immunocytofluorescence performed on the same cells indictated that the transgenic protein localized to the cell membrane (Fig. 3E). In order to confirm that the transgene localized correctly and functioned in vivo, we crossed TgVegfr 3 mice with $T b x 1^{\mathrm{Cre} /+}$ mice and analyzed expression of Vegfr3 (Fig. 3F) and phosphorylated ERK (pERK, Fig. 3G) in embryos at E15.5. We observed ectopic expression of the transgenic Vegfr3 protein in the tongue (Fig. 3F, a'), heart (Fig. 3F, b', c') and mandibular (masseter) muscle (Fig. 3G, a') in E15.5 Tbx ${ }^{\mathrm{Cre} /+}$; TgVegfr3 embryos but not in $\mathrm{Tb} \times \mathrm{I}^{\mathrm{Cre} /+}$ (control) embryos (Fig. 3F, a-c, Fig. 3G, a). Similarly, pERK was expressed in the masseter muscle

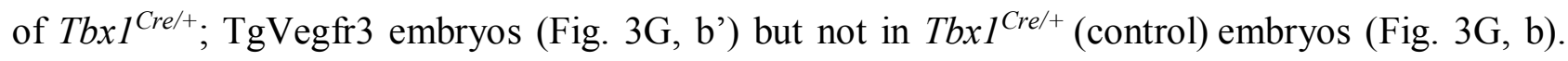
These results indicate that $T b x 1^{C r e}$ activates expression of transgenic Vegfr 3 in the endogenous $T b x 1$ expression domains and that the transgenic protein is able to activate the MAPK/ERK pathway, a known VegfC/Vegfr3 downstream signalling pathway that promotes cell proliferation.

\section{Vegfr3 over-expression partially rescues lymphatic defects in Tbx1-Vegfr3 compound mutants}

We tested whether the TgVegfr3 transgene was able to rescue the cardiac lymphatic vessel anomalies observed in $\mathrm{Tb} x \mathrm{I}^{\mathrm{Cre} /+}$; Vegfr $3^{f l o x /+}$ embryos. For this, we crossed $\mathrm{Tb} x \mathrm{I}^{\mathrm{Cre} /+}$ mice with TgVegfr3;Vegfr $3^{\text {flox/+ }}$ mice and analyzed cardiac lymphatic vessels in E18.5 embryos with the following genotypes: $\mathrm{Tbx} \mathrm{I}^{\mathrm{Cre} /+}, \mathrm{Tbx} \mathrm{I}^{\mathrm{Cre} /+} ; \mathrm{Vegfr} 3^{\text {flox/++}}, \mathrm{TgVegfr} 3 ; \mathrm{Tbx} \mathrm{I}^{\mathrm{Cre} /+}$; Vegfr $3^{\text {flox/+ }}$ (Fig. 4A and Table 1).

\begin{tabular}{|c|c|c|c|c|c|c|c|}
\hline & \multirow[b]{2}{*}{$\begin{array}{c}\text { Number } \\
\text { of } \\
\text { embryos }\end{array}$} & \multicolumn{2}{|c|}{ Number of vessels } & \multicolumn{2}{|c|}{ Length of vessels } & \multicolumn{2}{|c|}{ Width of vessels* } \\
\hline & & ventral & dorsal & ventral & dorsal & Ventral & dorsal \\
\hline WT & 5 & normal & normal & normal & normal & normal & normal \\
\hline $\operatorname{Tbx} 1^{\mathrm{Cre} /+}$ & 8 & normal & normal & normal & normal & normal & normal \\
\hline $\mathrm{Tbxl}^{\mathrm{Cre} /+} ; \mathrm{Vegfr} 3^{\text {flox } /+}$ & 8 & decreased & decreased & decreased & decreased & Increased & increased \\
\hline $\begin{array}{l}\text { TgVegfr } 3 ; \mathrm{Tbx} 1^{\mathrm{Cre} /+} \\
\text { Vegfr } 3^{\text {flox/+ }}\end{array}$ & 6 & $\#$ & decreased & $\#$ & normal & $\#$ & normal \\
\hline TgVegfr3; Tbx $1^{\mathrm{Cre} /+}$ & 5 & $\#$ & normal & $\#$ & normal & $\#$ & normal \\
\hline Tbx $1^{\text {Cre/lacZ }}$ & 3 & $\#$ & $\#$ & \# & \# & \# & $\#$ \\
\hline TgVegfr3;Tbx $1^{\text {CrellacZ }}$ & 4 & $\#$ & $\#$ & $\#$ & \# & $\#$ & $\#$ \\
\hline WT & 5 & normal & normal & normal & normal & normal & normal \\
\hline Vegfr $3^{+/-}$ & 5 & normal & normal & normal & normal & Increased & increased \\
\hline
\end{tabular}

Table 1. Summary of cardiac lymphatic phenotypes in E18.5 embryos for all genotypes analyzed.*, ratio length/area; \#, vessels absent in most embryos. 
Results showed that the transgene was able to rescue partially the cardiac lymphatic phenotype of compound heterozygotes, described in detail above and shown in Fig. 2C, c-c' and Fig. 4A, c, c'. Specifically, on the dorsal surface of the heart of TgVegfr3; Tbx ${ }^{\mathrm{Cre} /+} ; \mathrm{Vegfr} 3^{\text {flox/+ }}$ embryos (Fig. 4A, d'), the lymphatic vessel network was similar in appearance to that of $\mathrm{Tb}^{\mathrm{Cre} /+}$ (control) embryos (Fig. 4A, b'), being composed of mostly thin, well organized vessels, some of which extended to the apex of the heart. Quantitative analysis confirmed that the length and width of the dorsal lymphatic vessels was fully rescued in TgVegfr3; $\mathrm{Tbx} \mathrm{I}^{\mathrm{Cre} /{ }^{+}} ; \mathrm{Vegfr} 3^{f l o x /+}$ embryos (Fig. 4B, b', c'). In contrast, the number of lymphatic vessels was not rescued by the transgene (Fig. 4B, a') suggesting that their growth is regulated by $T b x 1$, consistent with our previously published findings (1). On the ventral surface of the same hearts, there was no appreciable rescue, in fact three out of five embryos lacked ventral lymphatic vessels, precluding a statistical analysis. These findings suggested that lymphatic vessel hypertrophy in $T b x 1^{\mathrm{Cre} /+} ; \mathrm{Vegfr} 3^{f l o x /+}$ embryos was due to reduced dosage of Vegfr 3 in ECs, and that the transgene was able to compensate for this in TgVegfr3; Tbx ${ }^{\mathrm{Cre} /+} ; \operatorname{Vegfr} 3^{\text {flox/+ }}$ embryos. Furthermore, as the number of lymphatic vessels was not rescued by the transgene, The different effects of the transgene on the dorsal versus ventral surface of the heart was puzzling initially, but became more clear when we analyzed TgVegfr3; $\mathrm{Tb}_{x} \mathrm{I}^{\mathrm{Cr} /+}$ embryos. In these mutants, the dorsal cardiac lymphatic vessels were indistinguishable from WT and $T b x 1^{\mathrm{Cre} /+}$ embryos while the ventral surface of the heart lacked lymphatic vessels (Supplementary Fig. S3). This suppressive effect of the transgene on ventral cardiac lymphangiogensis likely accounts for it's inability to rescue the ventral lymphatic hypoplasia caused by combined Tbx1-Vegfr3 haploinsufficiency..

As the transgene was quite effective at rescuing lymphatic anomalies on the dorsal surface of the heart, we tested whether it modified the cardiac lymphatic phenotype of Tbxl homozygous mutants (TgVegfr3;Tbx $1^{\text {Cre/lacZ }}$ ), with negative results (Table 1 and Supplementary Fig. S3).

In conclusion, we propose a model where Tbx1 and Vegfr3 interact in lymphatic ECs to establish a reciprocal gene dosage equilibrium that determines the correct number and morphology of sub-epicardial lymphatic vessels. The absence of lymphatic vessels on the ventral surface of the heart of different genetic mutants used in this study suggests that the Tbx1-Vegfr3 interaction is required prior to, or at the onset of cardiac lymphangiogenesis.

\section{DISCUSSION}

Our previous study indicated that $T b x 1$ regulates lymphatic development through the VegfCVegfr3 axis. In this study, we have demonstrated a strong genetic interaction between $T b x 1$ and 
$V e g f r 3$ that is critical for cardiac lymphangiogenesis and involves two distinct phenotypes, namely, the number of lymphatic vessels and lymphatic vessel morphology. In vivo rescue experiments indicated that these different aspects of the cardiac lymphatic phenotype are regulated independently by the two genes, with Tbx1 regulating the number of lymphatic vessels and Vegfr3 their morphology. We also found that for cardiac lymphatic development Vegfr3 is haploinsufficient, a feature that, to our knowledge, has not been reported before.

Rebalancing Vegfr3 gene dosage in Tbxl-deficient cells was not sufficient for normal cardiac lymphatic development, suggesting that there may be other effectors of Tbx1 function in lymphatic ECs. However, Tbx1 may also have a developmental function in EC progenitors that cannot be compensated by enhanced Vegfr3 expression. This hypothesis arises from the observation, reported here, that $T b x 1$-expressing cells and their descendants contribute extensively to the cardiac lymphatic network, and they are present in the earliest cardiac lymphatic vessels. Recently, it has been demonstrated that cardiac lymphatic vessels derive from venous and non-venous EC progenitors (2). In the future, it would be interesting to determine whether there is a contribution from the secondary heart field, where Tbxl is highly expressed, and where Tbxl-expressing multi-potent progenitors give rise to ECs that populate the aortic arch arteries and cardiac outflow tract (10), as well as to cardiomyocytes and branchiomeric muscles (15)(16).

We were intrigued by the different response of the ventral and dorsal surfaces of the heart to altered Vegfr3 gene dosage. We have shown here that the genetic interaction between Tbx 1 and Vegfr3 is especially evident on the ventral surface of the heart (Fig. 2A-c). Furthermore, the capacity of TgVegfr3 to rescue lymphatic anomalies in Tbxl-Vegfr3 compound heterozygotes was limited to the dorsal surface of the heart (Fig. 4d'). Finally, over-expression of Vegfr3 from the TgVegfr3 transgene had a strong effect on the ventral but not dorsal cardiac lymphatic vessels (Fig. 4e, 4e'). Note that in all three cases, a single copy of $T b x l\left(T b x l^{C r e /+}\right)$ was inactivated, which alone had no effect on cardiac lymphatics. Together, these results suggest that $T b x 1$ mutation sensitizes the ventral surface of the heart to altered $\operatorname{Veg} f r 3$ gene dosage (mild lymphatic hypertrophy in $\operatorname{Veg} f r 3^{+/}$mutants affects ventral and dorsal cardiac lymphatic vessels (Fig. 2C).

What might be the basis of this differential sensitivity? It is not likely to be linked to differences in activation of the transgene as this appeared to be uniform in the dorsal and ventral heart (Fig. 3F, b-c). In fact, the differential response was also evident in the absence of the transgene, in Tbx $1^{\text {Cre/+ }}$;Vegfr $3^{f l o x /+}$ compound heterozygotes. Other possibilities include, i) differences between lymphatic EC progenitors; Tbxl may be more highly expressed in ventral progenitors, ii) differences in vessel growth; Tbxl may be more highly expressed in ventral cardiac lymphatic vessels, and when it is absent they fail to grow, iii) differences in the local environment. Considering the first possibility, 
studies of early cardiac lymphatic vessel development have shown that they derive from an extracardiac source of Prox1+/Lyve1+ lymphatic ECs that are of mainly venous origin (cardinal vein), which reaches the heart at E12.5-E13 from the mediastinum on the dorsal surface of the heart and from the cardiac outflow tract on the ventral surface (17)(5)(2) (18). Whether these invading lymphatic ECs derive from different progenitors has not been reported. Here, we have shown that Tbxl-expressing cells and their descendants contribute extensively to both ventral and dorsal lymphatic networks suggesting that differential Tbxl expression in EC progenitors, or their descendants, does not account for the differential ventral $\gg$ dorsal sensitivity. Considering the second possibility, from E14.5, lymphatic vessels on the ventral and dorsal surfaces of the heart grow in a base-to-apex direction, extending more rapidly over the dorsal surface (2). If $T b x l$ were more highly expressed in ventral lymphatic vessels, or if there were a critical Tbxl function or Tbx1-Vegfr3 interaction in ventral lymphatic vessels, this might preferentially stall the growth of the ventral lymphatic network. Our results show that Tbxl is expressed in both ventral and dorsal lymphatic vessels at E14.5 (Fig. 1B-b', 1B-d'), but this does not exclude functional differences between the dorsal and ventral networks. Considering the third possibility, several studies have shown that the local molecular environment can profoundly influence Vegfr3 function in the context of blood vessel development. For example, it has been demonstrated that Vegfr3 may promote or antagonize angiogenesis through mechanisms that are Notch-dependent and VEGF-ligand-independent (19)(20). These mechanisms may also depend upon Tbxl dosage. As yet, there are no studies that address the issue in lymphatic vessels, but it is certainly possible that local differences in the molecular milieu on the ventral versus dorsal surfaces of the heart determine a greater sensitivity to the ventral network to altered Vegfr3 dosage. Finally, increased Vegfr3 expression in the endogenous domain or ectopic Vegfr3 expression may negatively affect cardiac lymphangiogenesis. We attempted to address this by generating TgVegfr3; Tie2 $2^{\text {Cre/+ }}$ embryos, but we did not recover any embryos with the desired genotype at E14.5, indicating that forcing expression of $V e g f r 3$ in all endothelial cells causes lethality prior to the onset of cardiac lymphangiogenesis. Evidently, reducing endogenous Vegfr 3 expression in TgVegfr3; Tbxl ${ }^{\mathrm{Cre} /+}$; Vegfr $3^{\text {flox/+ }}$ embryos is not sufficient to rescue cardiac lymphatic hypoplasia caused by expression of the transgene. This suggests that ectopic Vegfr 3 expression driven by Tbx ${ }^{C r e}$, for example in cardiomyocytes of the right ventricle, may be responsible for lymphatic hypoplasia in these mutants, perhaps by sequestering the ligand and thus reducing its availability to lymphatic ECs.

Intriguingly, recent clinical studies have linked VEGFR3 mutations to congenital heart disease (CHD), and the 22q11.2 deletion to lymphatic problems, encouraging speculation about a genetic interaction between $T B X 1$ and VEGFR3 in humans. In the first study, Jin et al. showed for the first time that VEGFR3 might play a critical role in heart development (21). In this study, exome 
sequencing of 2871 probands with CHD revealed dominant VEGFR3 mutations in $2.3 \%$ of cases of Tetralogy of Fallot (TOF), the most common CHD associated with 22q11.2DS, for which $T B X 1$ is the major disease gene. In the second study, Unolt et al. reported the presence of primary lymphedema in $4 / 1600(0.025 \%)$ patients with 22 q11.2DS (22). Prior to this, only a single case report had documented lymphatic abnormalities in a patient diagnosed with DiGeorge syndrome (23). The finding of primary lymphedema in 22q11.2DS patients supports the hypothesis of a TBX1-VEGFR3 interaction, through which $T B X 1$ heterozygosity predisposes to lymphatic anomalies by disrupting the VEGF/C-VEGFR3 signalling pathway. Considering the recent clinical findings and supporting data from mouse models of 22q11.2DS, it would be interesting to determine the frequency of deleterious VEGFR3 variants in 22q11.2DS patients, and establish whether this leads to more severe types of CHD.

\section{MATERIAL AND METHODS}

\section{Mouse lines and tissues}

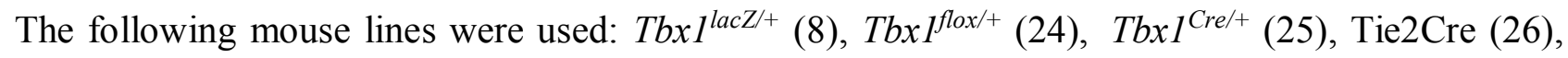
Vegfr $3^{f l o x /+}$ (14), R26R (12), Rosa ${ }^{m T m G}$ (13), TgCAGG-CreER ${ }^{\mathrm{TM}}$ (27). Genotyping of mice was performed according to the original reports. Activation of CAGG-CreER ${ }^{\mathrm{TM}}$ was performed with a single intraperitoneal injection of Tamoxifen (Sigma) at $75 \mathrm{mg} / \mathrm{kg}$ body weight at one of various time points between gestation days 10.5 and 16.5 .

\section{Salmon-gal staining, immunohistochemistry and immunofluorescence}

$\beta$-Gal activity was revealed by salmon-gal staining. Embryonic hearts at E14.5, E15.5 and E18.5 were isolated and fixed for $30 \mathrm{~min}$ in glutaraldehyde $(0.2 \%)$, paraformaldehyde $(2 \%)$, EGTA (5 mM), magnesium chloride $(2 \mathrm{mM})$ and phosphate buffer a pH $7.3(0.1 \mathrm{M})$, then washed three times $(20$ mins per wash) in a solution containing sodium deoxycholate (0.1\%), NP40 (0.2\%), magnesium chloride $(2 \mathrm{mM})$ and phosphate buffer $\mathrm{pH} 7.3(0.1 \mathrm{M})$. Hearts were stained in a solution containing salmon-gal $(1 \mathrm{mg} / \mathrm{mL})$ and NBT $(0.4 \mathrm{mM})$ in washing solution in the dark at $37^{\circ} \mathrm{C}$ with gentle rotation until the desired staining was achieved. Immunostaining was performed using the following primary antibodies: rat anti-Vegfr3 (eBioscience), rabbit anti-Lyve1 (Abcam), rabbit anti-GFP (Invitrogen), mouse anti-pERK (Cell Signalling), diluted 1: 400, and secondary antibodies: HRP-conjugated goat anti-rat IgG (Kirkegaard \& Perry Laboratories), HRP-conjugated anti-rabbit IgG (GE Healthcare), diluted 1:200. Fluorescent antibodies were visualized using Vectashield imaging medium (Vector Laboratories). Non-fluorescent antibodies were visualized using DAB (Vector Laboratories). Whole- 
mount specimens were photographed using a dissecting microscope (Stemi 2000; Carl Zeiss, Inc.) equipped with a camera (Axiocam; Carl Zeiss, Inc.) and the manufacturer's acquisition software. Images were acquired at 28x magnification.

\section{Quantitative analysis of lymphatic vessel anomalies}

We analyzed a minimum of 5 and a maximum of 8 embryos per genotype in all experiments. Threedimensional images were digitally reconstructed from $z$ stacks. For each image, we manually counted all the lymphatic vessels on the dorsal and ventral surfaces of the heart, and we measured the length and area of each vessel. We then calculated the ratio length/area to determine the width of each single vessel. Quantitative analysis was performed using the ImageJ software, (www.uhnresearch.ca/facilities/wcif/imagej).

\section{Statistical analysis}

We used the non-parametric Kruskall-Wallis test for the analysis of lymphatic vessels in all experiments with a single exception, namely $\operatorname{Veg} f r 3^{+/-}$vs WT embryos, for which the Mann- Whitney $\mathrm{U}$ test was used. We first calculated the mean number of vascular features (number, length, area/length vessels) for each embryo. We then calculated the mean value for the group (same genotype). The latter value was used for the statistical analysis.

\section{Generation of transgenic mouse embryonic stem cells}

The starting vector was the pCIG2 plasmid (28) without the nuclear localization sequence of EGFPCDS that consists of a CMV-IE enhancer, chicken $\beta$-actin promoter, MCS, IRES-GFP and rabbit $\beta$ globin polyA. A loxP-flanked neomycin resistance cassette was cloned downstream of the $\beta$-actin promoter followed by a full-length mouse Vegfr3 cDNA (GeneCoepia). The linearized Vegfr3 transgenic construct, named TgVegfr3, was electroporated into feeder-free E14Tg2A.4 mouse ES cells using the ECM 630 Electro Cell Manipulator System (BTX). Twentyfour hours postelectroporation, the cells were selected in G418 at a final concentration of $250 \mu \mathrm{g} / \mathrm{ml}$. ES cell clones were picked 12 day after plating.

\section{Identification of correctly targeted ES cell clones by southern blotting}

Two probes were generated for southern blotting experiments. The first probe distinguished the transgene from the endogenous $\operatorname{Veg} f r 3$ gene. It was generated by PCR amplifying a segment of exon 13 of the Vegfr3 cDNA, using the following primers: pF 5'CTTAGAAGGCCAGTCCGTGC 3', pR 5'CCTGCACGGACAGGTACTTC 3'. BamHI digestion of genomic DNA from ES clones generated a $2.5 \mathrm{~Kb}$ fragment in the presence of the transgene and a $2.8 \mathrm{~Kb}$ fragment in the endogenous Vegfr 3 
gene. To identify clones with a single insertion of the transgene, a second probe was generated as follows; a region of the neomycin resistance cassette was amplified by PCR using the primers: $\mathrm{pF} 5$ ' GCACAACAGACAATCGGCTG 3', pR 5'GATACTTTCTCGGCAGGAGC 3'. This probe, which was designed upstream the first BamHI cutting site in the linearized TgVegfr3 construct, localized within the neomycin-resistance cassette. The first BamHI cutting site upstream of the region amplified localized to genomic DNA, and thus its' position depended upon the insertion site of the electroporated TgVegfr 3 construct. Thus, the size of the fragment recognized by this probe varied from clone to clone, and within in the same clone, from insertion to insertion, in the case of multiple integrations of the transgene. ES cell clones containing a single copy of TgVegfr3 were analyzed by PCR to confirm that the entire Vegfr3 cDNA was present, using the primers: $\mathrm{pF}$ 5'CGACGAATtCGGtACCATGCAG 3', pR 5'GCACGGACTGGCCTTCTAAG 3'. These primers amplified a region extending from $16 \mathrm{bp}$ upstream of the ATG to $20 \mathrm{bp}$ downstream of the BamHI site. A 1741 bp fragment was expected.

\section{Generation of transgenic mice}

Two correctly targeted ES cell clones were selected for microinjection into mouse blastocysts. Four males chimeras were obtained. These males were bred with black C57BL/6 female mice and three germ line transmissions were obtained. These founders were crossed into the C57BL/6 strain. Mice were genotyped by PCR using DNA extracted from tail biopsies, using following primer pairs: F-5'ATCGACCTGGCAGACTCCAA-3'; R-5'-GAAAACTGCGATGACGCCAGT-3'.

PCR products were separated on $2 \%$ agarose gels.

\section{Western blot analysis}

Transfected C2C12 cells were collected 24h after transfection, washed with PBS and lysed in lysis buffer (TrisHCl 20mM pH7.4, NaCl 100mM, 10mM MgCl2, NP-40 1X, Glycerol 10\%, proteases inibitors). Denatured proteins were separated by SDS-PAGE, transferred to Immuno-Blot PVDF Membrane (BioRad) for protein blotting, Membranes were blocked for $1 \mathrm{~h}$ at RT in TBST (150 mM $\mathrm{NaCl}, 10 \mathrm{mM}$ Tris- $\mathrm{HCl}, \mathrm{pH} 7.4$, and $0.05 \%$ Tween) and powdered milk. The membranes were incubated overnight at $4^{\circ} \mathrm{C}$ in primary antibodies diluted in TBST-5\% milk, then for $1 \mathrm{~h}$ at RT with horseradish peroxidase-conjugated secondary antibodies (diluted in TBST-5\% milk). Protein binding was detected by ECS (Amersham) using Hyperfilm (Amersham). The molecular masses of proteins were estimated relative to the electrophoretic mobility of the co-transferred, pre-stained protein marker All Precision Blue (Biorad). The primary antibodies used were; rat anti-Vegfr3 (eBioscience), rabbit anti-GFP (Sigma-Aldrich). 


\section{Immunofluorescence on $\mathrm{C} 2 \mathrm{C} 12$ cells}

$\mathrm{C} 2 \mathrm{C} 12$ were plated into 6 -well plates on coverslips. The next day, cells were co-transfected with the TgVegfr3 construct and Cre recombinase vector. Immunocytofluorescence was performed 24 hours after transfection. Cells were washed and fixed with 3\% paraformaldehyde/PBS for 20 min at RT, permeabilized with $0.1 \%$ Triton X-100/PBS for 25 min and blocked with 1\% BSA/PBS for 45 min. Cells were incubated with anti-Vegfr3 antibody (eBioscience) diluted 1:100 in 0,1\% BSA/PBS for 3 hours, washed 3 times with PBS, incubated for $1 \mathrm{hr}$ with secondary antibody AlexaFluor anti-rat 494nm (Invitrogen) 1:200 in PBS. The fluorescent antibody was visualized using imaging medium (Vectashield with DAPI, Vector Laboratories). For visualization, the coverslip was positioned on a slide and images were acquired using confocal microscopy.

\section{ACKNOWLEDGEMENTS}

We are grateful for the support provided by the Integrated Microscopy Core and the Animal Facility at the Institute of Genetic and Biophysics 'ABT'/CNR, Naples. Vegfr $3^{\text {flox }}$ mice were kindly provided by Dr. Kari Alitalo.

\section{CONFLICT OF INTEREST}

None

\section{FUNDING}

This work was supported by grants from the Fondation Leducq TNE 15CVD01, to E.I. and to A.B., and from the Italian Ministry of Health (RF-2011-02347197) to A.B. M.G.T was supported by a doctoral fellowship from the European School of Molecular Medicine (SEMM). 


\section{REFERENCES}

1. Chen,L., Mupo,A., Huynh,T., Cioffi,S., Woods,M., Jin,C., McKeehan,W., Thompson-Snipes,L., Baldini,A. and Illingworth,E. (2010) Tbx1 regulates Vegfr3 and is required for lymphatic vessel development. J. Cell Biol., 189, 417-424.

2. Klotz,L., Norman,S., Vieira,J.M., Masters,M., Rohling,M., Dubé,K.N., Bollini,S., Matsuzaki,F., Carr,C.A. and Riley,P.R. (2015) Cardiac lymphatics are heterogeneous in origin and respond to injury. Nature, 522, 62-67.

3. Henri,O., Pouehe,C., Houssari,M., Galas,L., Nicol,L., Edwards-Lévy,F., Henry,J.-P., Dumesnil,A., Boukhalfa,I., Banquet,S., et al. (2016) Selective Stimulation of Cardiac Lymphangiogenesis Reduces Myocardial Edema and Fibrosis Leading to Improved Cardiac Function Following Myocardial Infarction. Circulation, 133, 1484-1497; discussion 1497.

4. Ishikawa,Y., Akishima-Fukasawa,Y., Ito,K., Akasaka,Y., Tanaka,M., Shimokawa,R., KimuraMatsumoto,M., Morita,H., Sato,S., Kamata,I., et al. (2007) Lymphangiogenesis in myocardial remodelling after infarction. Histopathology, 51, 345-353.

5. Flaht-Zabost,A., Gula,G., Ciszek,B., Czarnowska,E., Jankowska-Steifer,E., Madej,M., NiderlaBielińska,J., Radomska-Leśniewska,D. and Ratajska,A. (2014) Cardiac mouse lymphatics: developmental and anatomical update. Anat Rec (Hoboken), 297, 1115-1130.

6. Kataru,R.P., Jung,K., Jang,C., Yang,H., Schwendener,R.A., Baik,J.E., Han,S.H., Alitalo,K. and Koh,G.Y. (2009) Critical role of CD11b+ macrophages and VEGF in inflammatory lymphangiogenesis, antigen clearance, and inflammation resolution. Blood, 113, 5650-5659.

7. Huggenberger,R., Siddiqui,S.S., Brander,D., Ullmann,S., Zimmermann,K., Antsiferova,M., Werner,S., Alitalo,K. and Detmar,M. (2011) An important role of lymphatic vessel activation in limiting acute inflammation. Blood, 117, 4667-4678.

8. Lindsay,E.A., Vitelli,F., Su,H., Morishima,M., Huynh,T., Pramparo,T., Jurecic,V., Ogunrinu,G., Sutherland,H.F., Scambler,P.J., et al. (2001) Tbx1 haploinsufficieny in the DiGeorge syndrome region causes aortic arch defects in mice. Nature, 410, 97-101.

9. Kaipainen,A., Korhonen,J., Mustonen,T., van Hinsbergh,V.W., Fang,G.H., Dumont,D., Breitman,M. and Alitalo,K. (1995) Expression of the fms-like tyrosine kinase 4 gene becomes restricted to lymphatic endothelium during development. Proc Natl Acad Sci U S A, 92, 3566-70.

10. Xu,H., Cerrato,F. and Baldini,A. (2005) Timed mutation and cell-fate mapping reveal reiterated roles of Tbx 1 during embryogenesis, and a crucial function during segmentation of the pharyngeal system via regulation of endoderm expansion. Development, 132, 4387-95.

11. Théveniau-Ruissy,M., Pérez-Pomares,J.-M., Parisot,P., Baldini,A., Miquerol,L. and Kelly,R.G. (2016) Coronary stem development in wild-type and Tbx1 null mouse hearts. Dev. Dyn., 245, 445459.

12. Soriano,P. (1999) Generalized lacZ expression with the ROSA26 Cre reporter strain. Nat Genet, 21, 70-1. 
13. Muzumdar,M.D., Tasic,B., Miyamichi,K., Li,L. and Luo,L. (2007) A global double-fluorescent Cre reporter mouse. Genesis, 45, 593-605.

14. Zarkada,G., Heinolainen,K., Makinen,T., Kubota,Y. and Alitalo,K. (2015) VEGFR3 does not sustain retinal angiogenesis without VEGFR2. Proc. Natl. Acad. Sci. U.S.A., 112, 761-766.

15. Tirosh-Finkel,L., Elhanany,H., Rinon,A. and Tzahor,E. (2006) Mesoderm progenitor cells of common origin contribute to the head musculature and the cardiac outflow tract. Development, 133, 1943-1953.

16. Lescroart,F., Kelly,R.G., Le Garrec,J.-F., Nicolas,J.-F., Meilhac,S.M. and Buckingham,M. (2010) Clonal analysis reveals common lineage relationships between head muscles and second heart field derivatives in the mouse embryo. Development, 137, 3269-3279.

17. Flaht,A., Jankowska-Steifer,E., Radomska,D.M., Madej,M., Gula,G., Kujawa,M. and Ratajska,A. (2012) Cellular phenotypes and spatio-temporal patterns of lymphatic vessel development in embryonic mouse hearts. Dev. Dyn., 241, 1473-1486.

18. Norman,S. and Riley,P.R. (2016) Anatomy and development of the cardiac lymphatic vasculature: Its role in injury and disease. Clin Anat, 29, 305-315.

19. Tammela,T., Zarkada,G., Nurmi,H., Jakobsson,L., Heinolainen,K., Tvorogov,D., Zheng,W., Franco,C.A., Murtomaki,A., Aranda,E., et al. (2011) VEGFR-3 controls tip to stalk conversion at vessel fusion sites by reinforcing Notch signalling. Nature cell biology, 13, 1202-13.

20. Benedito,R., Rocha,S.F., Woeste,M., Zamykal,M., Radtke,F., Casanovas,O., Duarte,A., Pytowski,B. and Adams,R.H. (2012) Notch-dependent VEGFR3 upregulation allows angiogenesis without VEGF-VEGFR2 signalling. Nature, 10.1038/nature10908.

21. Jin,S.C., Homsy,J., Zaidi,S., Lu,Q., Morton,S., DePalma,S.R., Zeng,X., Qi,H., Chang,W., Sierant,M.C., et al. (2017) Contribution of rare inherited and de novo variants in 2,871 congenital heart disease probands. Nat. Genet., 49, 1593-1601.

22. Unolt,M., Barry,J., Digilio,M.C., Marino,B., Bassett,A., Oechslin,E., Low,D.W., Belasco,J.B., Kallish,S., Sullivan,K., et al. (2018) Primary lymphedema and other lymphatic anomalies are associated with 22q11.2 deletion syndrome. Eur J Med Genet, 61, 411-415.

23. Mansir,T., Lacombe,D., Lamireau,T., Taine,L., Chateil,J.F., Le Bail,B., Demarquez,J.L. and Fayon,M. (1999) Abdominal lymphatic dysplasia and 22q11 microdeletion [In Process Citation]. Genet Couns, 10, 67-70.

24. Xu,H., Morishima,M., Wylie,J.N., Schwartz,R.J., Bruneau,B.G., Lindsay,E.A. and Baldini,A. (2004) Tbx1 has a dual role in the morphogenesis of the cardiac outflow tract. Development, 131, $3217-27$.

25. Huynh,T., Chen,L., Terrell,P. and Baldini,A. (2007) A fate map of Tbx1 expressing cells reveals heterogeneity in the second cardiac field. Genesis, 45, 470-5.

26. Kisanuki,Y.Y., Hammer,R.E., Miyazaki,J., Williams,S.C., Richardson,J.A. and Yanagisawa,M. 
(2001) Tie2-Cre transgenic mice: a new model for endothelial cell-lineage analysis in vivo. Dev Biol, 230, 230-42.

27. Hayashi,S. and McMahon,A.P. (2002) Efficient recombination in diverse tissues by a tamoxifen-inducible form of Cre: a tool for temporally regulated gene activation/inactivation in the mouse. Dev Biol, 244, 305-18.

28. Megason,S.G. and McMahon,A.P. (2002) A mitogen gradient of dorsal midline Wnts organizes growth in the CNS. Development, 129, 2087-2098. 


\section{FIGURE LEGENDS}

\section{$\underline{\text { Figure } 1}$}

Tbxl and Vegfr3 are co-expressed in cardiac lymphatic vessels. (A) $\beta$-gal staining of E18.5 Tbx $1^{\text {lacZ/+ }}$ hearts shows that $T b x l$ is expressed in the conotruncus (arrow in a) and in cardiac lymphatic vessels (a-a'), identified in b-b' by anti-VEGFR3 immunostaining. Fate mapping of Tbxl-expressing cells and their descendents in $T b x 1^{c r e /+;} \operatorname{Rosa}^{m T-m G}$ hearts (c-c') reveals a contribution to cardiac lymphatic vessels and to the right ventricular myocardium. (B). Vegfr3 immunostaining (left) of E.14.5 (a-d) and E.15.5 (e-h) Tbx $1^{\text {Cre/+ }}$ hearts reveals early cardiac lymphatic vessel development (arrows in a', c', e', g'). $\beta$-gal staining (right) of E.14.5 (b, b', d, d') and E.15.5 (f, f', h, h') Tbx ${ }^{\text {lacZ/+ }}{ }^{\text {hearts shows }}$ that $T b x l$ is expressed in lymphatic vessels at the base of the outflow tract ventrally (arrows in b', f') and in the sinus venosus dorsally (arrows in d', h'). Temporal requirement of Tbxl for cardiac lymphatic vessel development (C). Cardiac lymphatic vessels revealed by Vegfr3 immunostaining of E18.5 hearts following Tamoxifen (TM) induced inactivation of Tbxl between E10.5 and E16.5 (Fig. $1 \mathrm{C}, \mathrm{b}-\mathrm{f}$ ) shows that Tbx1 is required for cardiac lymphangiogenesis before E14.5. Scale bar $100 \mu \mathrm{M}$ Abbreviations: LV, left ventricle, RV, right ventricle, SV, sinus venosus, OFT, outflow tract.

\section{Figure 2}

Genetic interaction Tbx1-Vegfr3 in cardiac lymphatic development. (A, C) Lyve-1 immunostaining identifies sub-epicardial lymphatic vessels in rappresentative hearts of E18.5 embryos of the genotypes shown (punctate signal derives from Lyve1+ macrophages). (A) Arrows in c, c' indicate abnormal cardiac lymphatic vessels in compound heterozygous embryos. B) Quantitative analysis revealed a reduced number (a-a') reduced length (b-b') and increased width (c-c') of ventral and dorsal cardiac lymphatic vessels in E18.5 $\mathrm{Tbx} \mathrm{I}^{\mathrm{Cre} /{ }^{+}} ; \mathrm{Veg}$ fr $3^{\text {flox/+ }}$ embryos compared to WT (a-a') and Tbx $1^{\text {Cre/+ }}$ (control) embryos (b-b'). C) Vegfr3 haploinsufficiency in cardiac lymphatic development. Lyve-1 immunostaining reveals mild hypertrophy of cardiac lymphatic vessels in E18.5 $\mathrm{Vegfr} \mathrm{f}^{\mathrm{ft}-}$ embryos (b-b'). D) Quantitative analysis (D) shows that number (a-a') and length (b-b') of cardiac lymphatic vessels were normal, but they were moderately dilated (c-c'). The cardiac Abbreviations: $\mathrm{LV}$, left ventricle, $\mathrm{RV}$, right ventricle. ${ }^{*} \mathrm{P}<0.05,{ }^{* *} \mathrm{P} \leq 0.01,{ }^{* * *} \mathrm{P} \leq 0.001$. Error bars (SEM).

\section{Figure 3}

Generation and in vivo expression of the Cre-inducible Vegfr3 transgene. (A) Construct used to generate TgVegfr3 transgenic mouse. (B) Southern blotting with the neomycin probe showing 2 out of 22 clones that carried a single copy of the transgene. (C) Southern blotting with the Vegfr3 probe confirmed the identity of the transgene $(2.5 \mathrm{~Kb})$, compared to the endogenous gene $(2.8 \mathrm{~Kb})$. Clones D9 and E8 were injected into mouse blastocysts. (D, upper panel) Western blot analysis with antiVEGFR3 revealed that the transgenic VEFGR3 protein was full length. Proteins were resolved on an 8\% SDS-polyacrylamide gel. Lane 1: P19 cells (positive control) express Vegfr3. Lane 2: C2C12 cells (negative control) do not express Vegfr3). Lane 3: C2C12 + empty vector. Lane 4: C2C12+ 
pCIG2-Vegfr3 plasmid (clone 1). Lane 5: C2C12 + pCIG2-Vegfr3 plasmid (clone 2). In lanes 1 and 4 show three bands corresponding to the full length protein (195KDa, red arrows), the pre-peptide (175KDa, blue arrows) and the proteolytically cleaved form (125 KDa band green arrows). (D, lower panel). The same protein samples probed with anti-GFP. Lanes 3, 4 and 5 show the GFP protein product confirming Cre activated of Vegfr3 and GFP expression. (E) shows Vegfr3 protein localization in $\mathrm{C} 2 \mathrm{C} 12$ cells co-transfected with a selected Vegfr3 construct and a construct expressing Cre recombinase. Immunofluorescence was performed 24 hours after transfection: a) DAPI staining of cell nuclei, b) Anti-Vegfr3 staining shows that the transgenic Vegfr3 protein localized correctly to the membrane, c) Merged image of a) and b). (F) Vegfr3 protein production and localization in transgenic mouse. Immunohistochemistry with anti-Vegfr 3 antibody on coronal sections of the head of E15.5 Tbx $1^{\mathrm{Cre} /+}$ (a) and TgVegfr3; $\mathrm{Tb}_{\mathrm{C}} \mathrm{I}^{\mathrm{Cre} /+}$ (b) embryos showed ectopic Vegfr3 protein expression in the tongue of TgVegfr3;Tbxl ${ }^{\text {Cre/+ }}$ embryos (b). Hearts of E18.5 Tbx $1^{\text {Cre/+ }}$ (b, c) and TgVegfr3;Tbx $1^{\text {Cre/+ }}$ (b', c') embryos showed ectopic Vegfr3 protein expression in the ventral and dorsal myocardium. (G) Immunohistochemistry with anti-Vegfr3 antibody (a, a') and anti-pERK antibody (b, b') on coronal sections of the head of E18.5 Tbx1 ${ }^{\mathrm{Cre} /+}$ (a, b) and TgVegfr3;Tbx1 ${ }^{\mathrm{Cre} /+}$ (a', b') embryos showed ectopic expression of both proteins in the masseter muscle in TgVegfr3; Tbx ${ }^{\mathrm{Cre} /+}$ embryos.

\section{Figure 4}

Vegfr 3 expression from the TgVegfr 3 transgene partially rescues cardiac lymphatic vessel anomalies in Tbxl-Vegfr3 compound heterozygotes. (A) Lyve-1 immunostaining identifies cardiac lymphatic vessels in all genotypes shown. Hearts of E18.5 TgVegfr3; Tbx $1^{\mathrm{Cre} /+} ; \operatorname{Vegfr} 3^{\text {flox/++}}$ embryos (d-d') reveal partial rescue of lymphatic anomalies observed in $T b x 1^{\mathrm{Cre} /+} ; \mathrm{Veg} f \mathrm{r} 3^{\text {flox/+ }}$ embryos (c-c'). Quantitative analysis (B) showed rescue of vessel length (b') and width (c') but not vessel number (a') on the dorsal surface of the heart of $T g V e g f r 3 ; T b x 1^{\text {Cre/+ }} ; \operatorname{Vegfr} 3^{f l o x /+}$ embryos compared to $T b x 1^{C r e /+}$; Vegfr $3^{f l o x}$ embryos (c, c'). There was no rescue of ventral lymphatic vessels. Abbreviations: $\mathrm{LV}$, left ventricle, $\mathrm{RV}$, right ventricle. ${ }^{*} \mathrm{P}<0.05,{ }^{* *} \mathrm{P} \leq 0.01,{ }^{* * *} \mathrm{P} \leq 0.001$. Error bars (SEM), \#, vessels absent in most TgVegfr 3 ; Tbx $1^{\mathrm{Cre} /+} ;$ Vegfr $3^{\text {flox/+ }}$ embryos (statistical analysis not performed).

\section{SUPPLEMENTARY FIGURE LEGENDS}

\section{Supplementary Figure S1}

Tbx1-expressing cells and their descendants populate cardiac lymphatic vessels. $\beta$-gal staining of E18.5 $\mathrm{Tb}^{\mathrm{Cre} / \mathrm{+}}$; R26R hearts (a) and in transverse sections of the heart (a') shows that Tbxl is expressed in cardiac lymphatic vessels, identified by anti-Vegfr3 immunohistochemistry in c, c' c', and in myocardium of the right ventricle. 
Genetical interaction Tbxl-Vegfr3 in intestinal lymphatic development. Lyve-1 immunostaining identifies lymphatic vessels in the intestinal tract of E18.5 embryos of all genotypes shown. White arrows indicate lymphatic vessels in the intestinal wall and in the membranous mesentery.

\section{Supplementary Figure S3}

Forced expression of Vegfr3 suppresses ventral cardiac lymphangiogenesis on a Tbxl heterozygous background. Lyve-1 immunostaining identifies cardiac lymphatic vessels in all genotypes shown. A. Hearts of E18.5 TgVegfr3; Tbx1 ${ }^{\mathrm{Cre} /+}$ embryos show normal cardiac lymphatic vessels on the dorsal surface and absence of these vessels on the ventral surface. B. Tbxl homozygous embryos (a, a') lack cardiac lymphatic vessels. Increasing Vegfr3 expression in Tbxl-deficient cells via $\mathrm{Tb}_{\mathrm{C}} \mathrm{Cre}_{-}$ mediated activation of the transgene does not modify this phenotype (b, b'). 


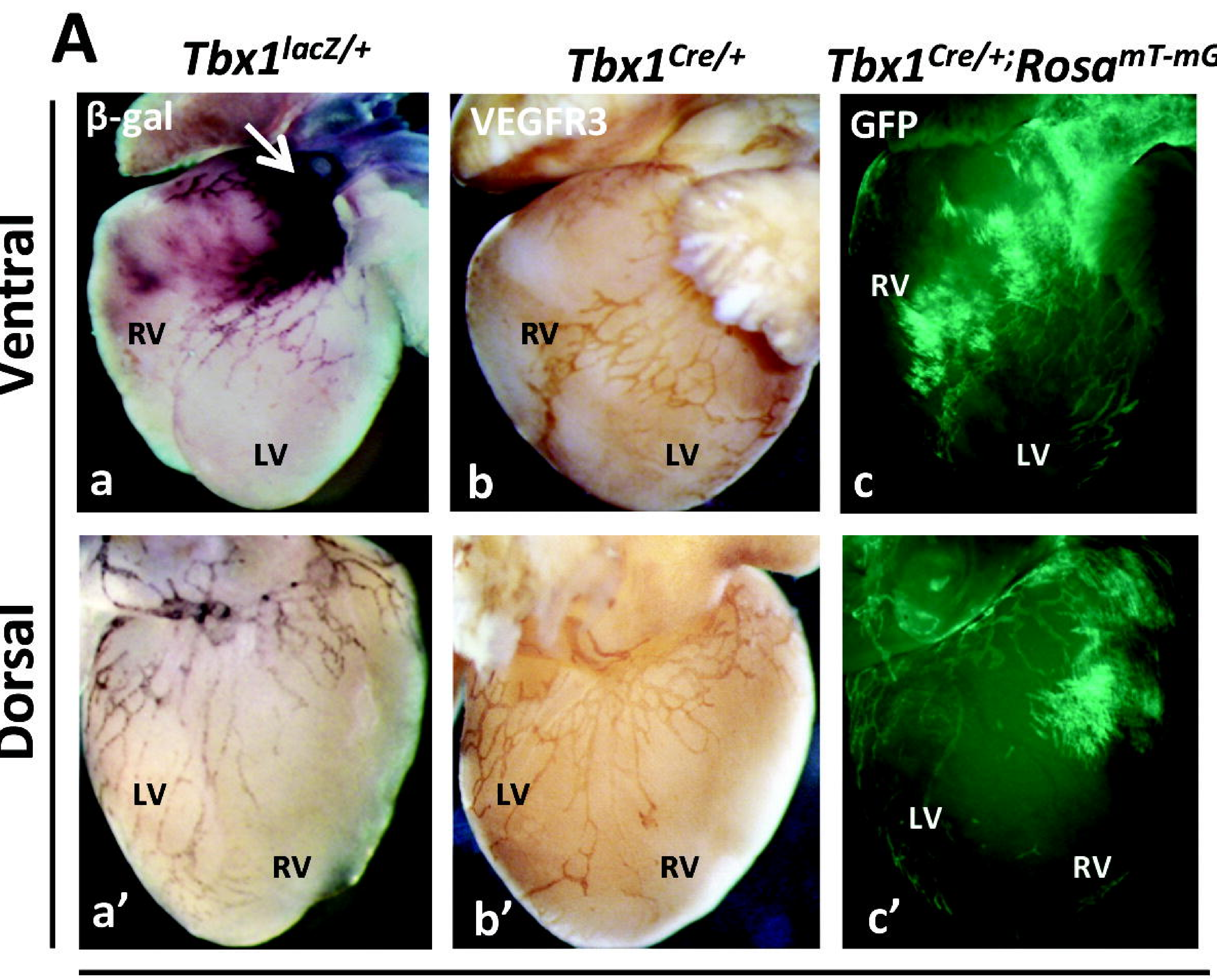

E 18.5

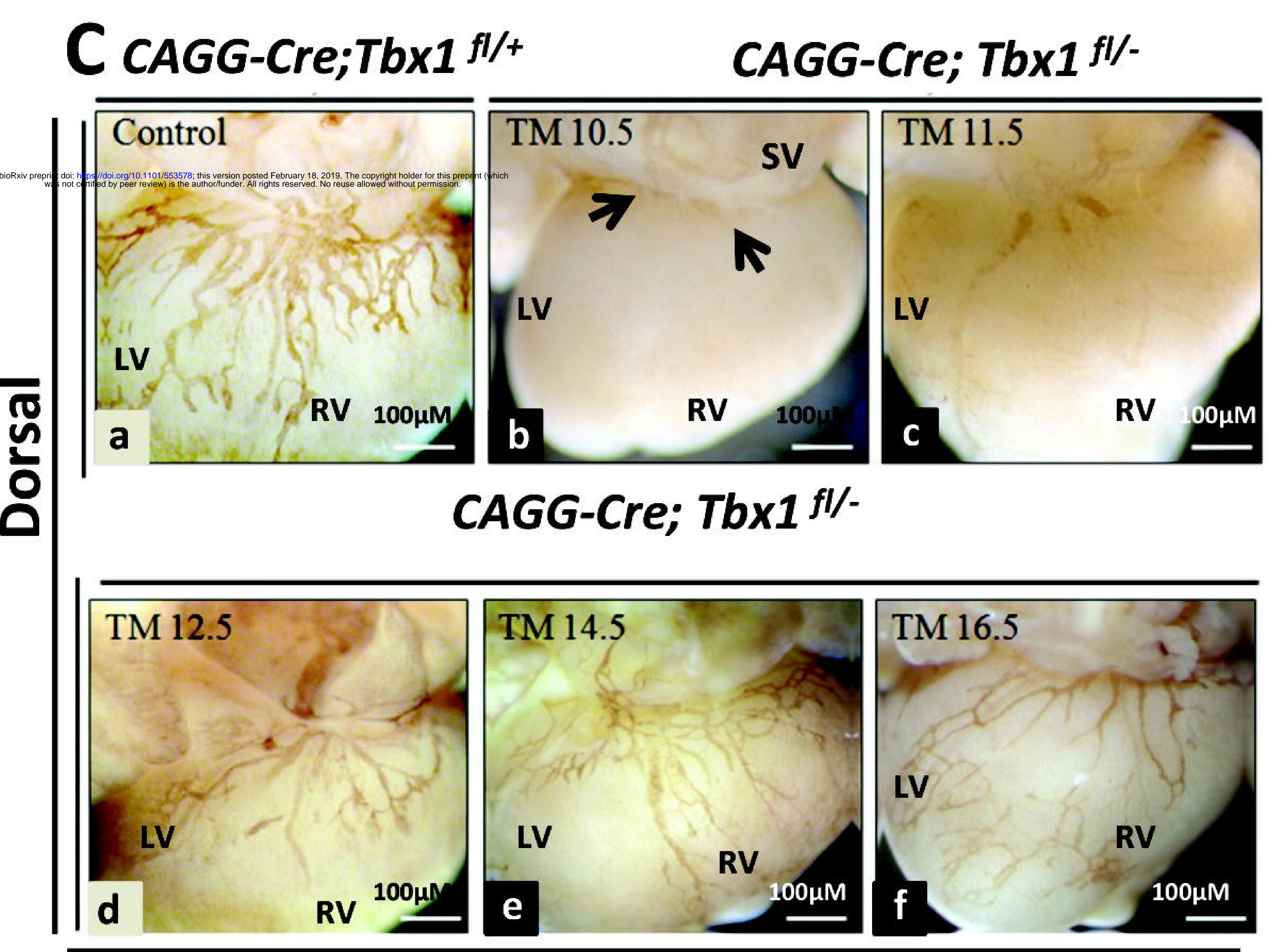

Vegfr3
B

Tbx $1^{\mathrm{Cre} /+}$
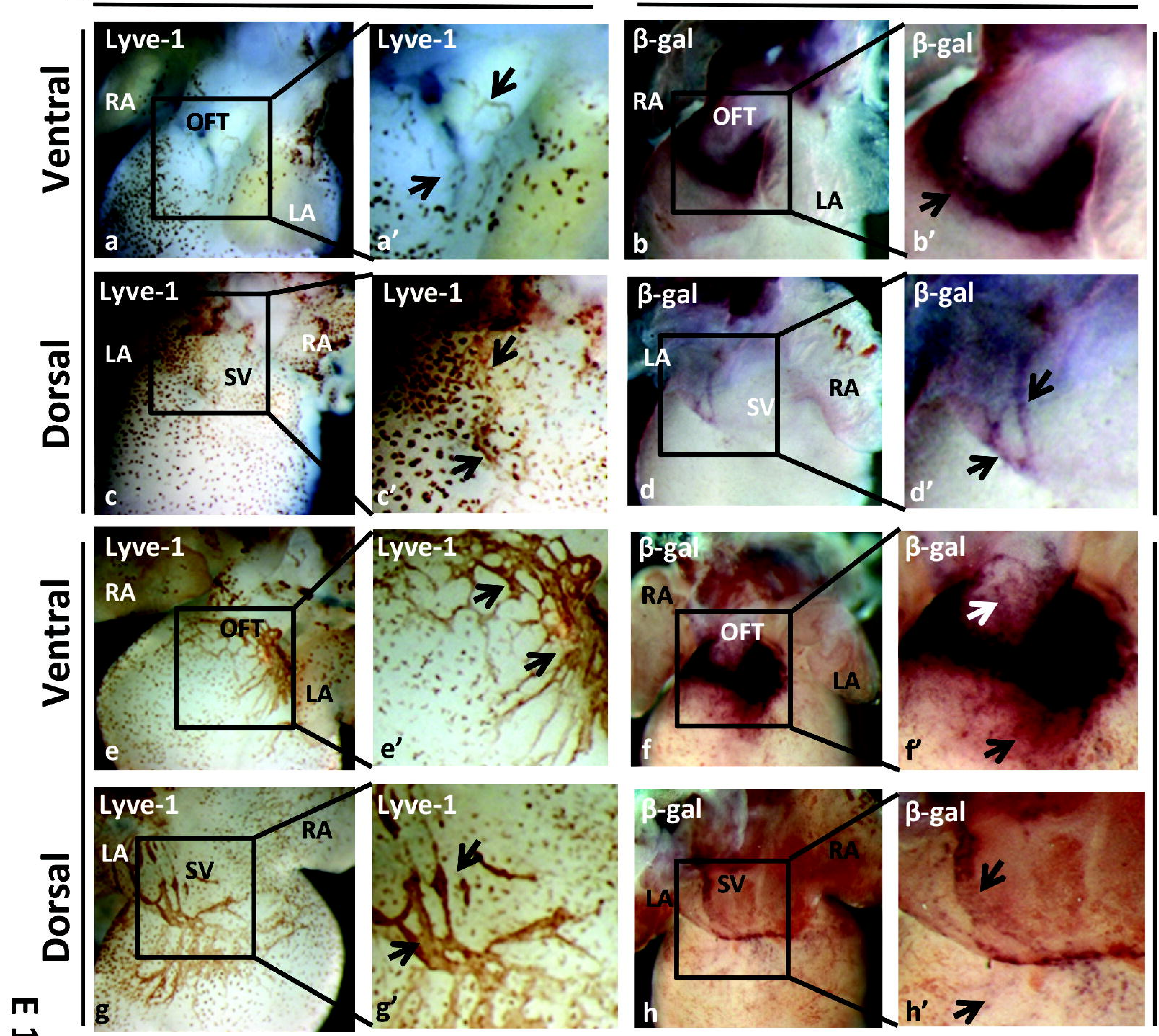

$\infty$
$\infty$
$v$

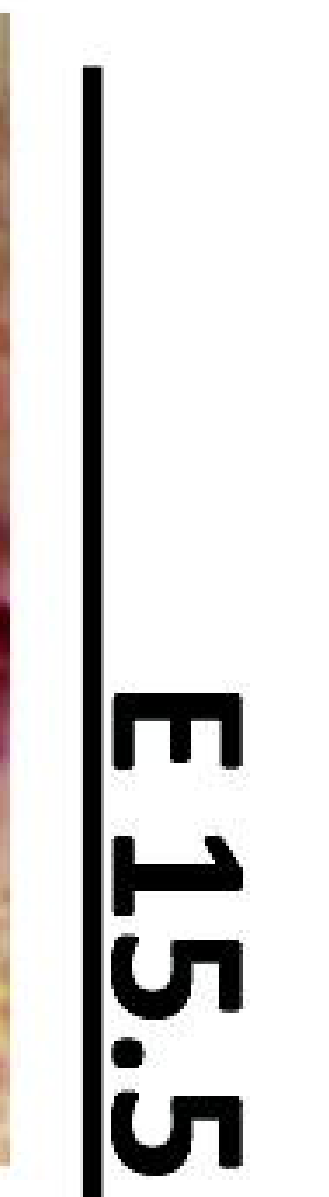
vi 


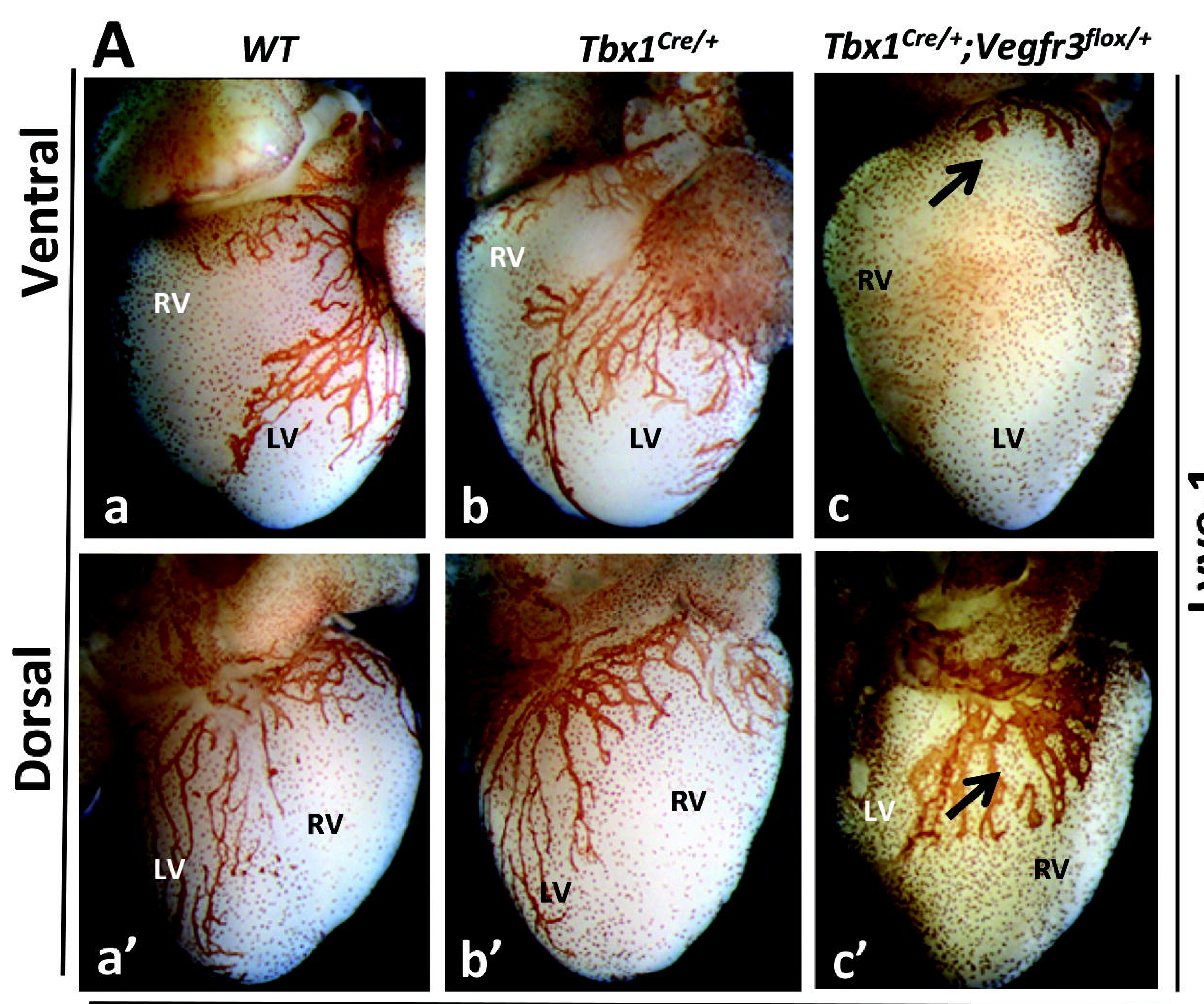

E 18.5
B

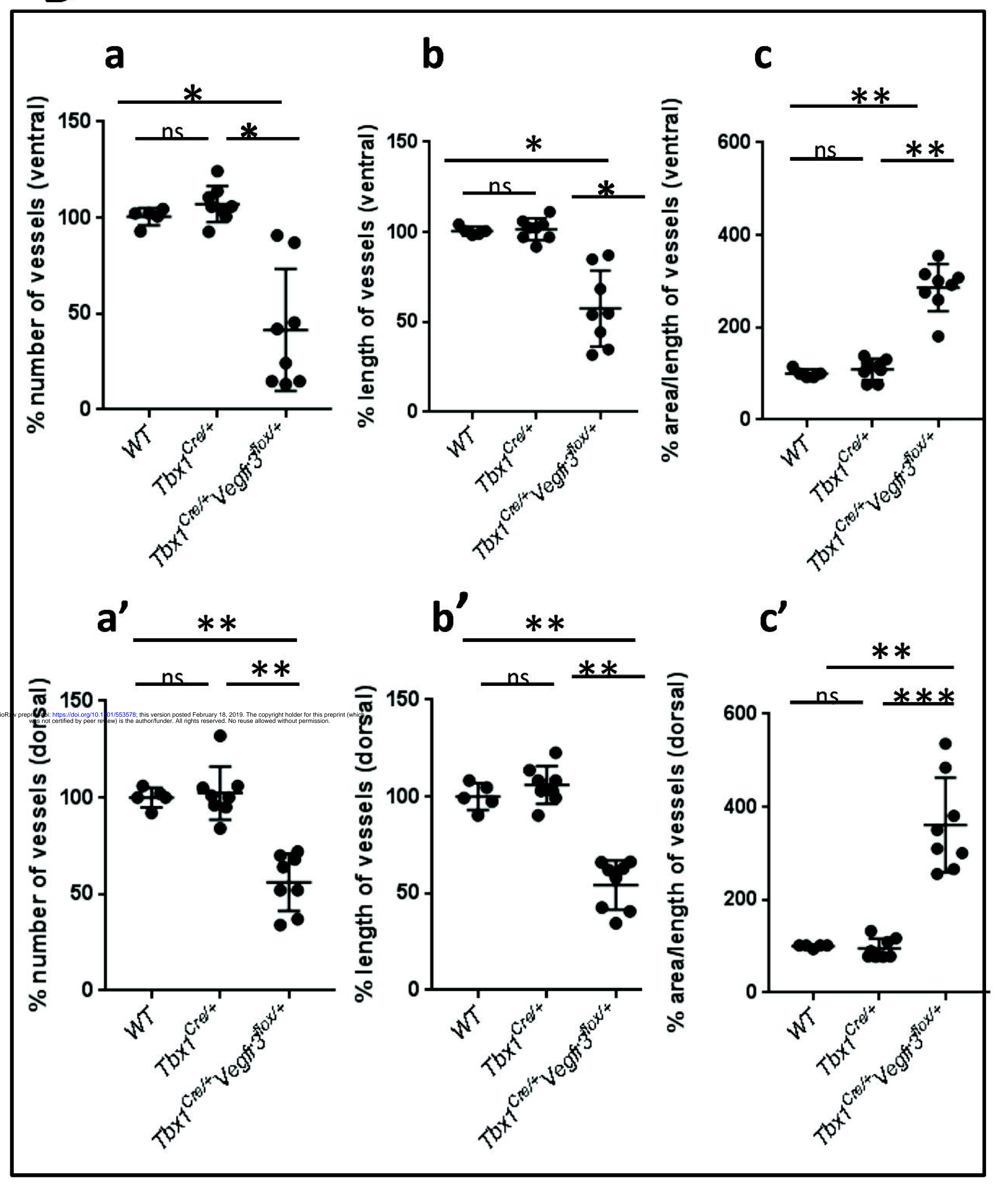

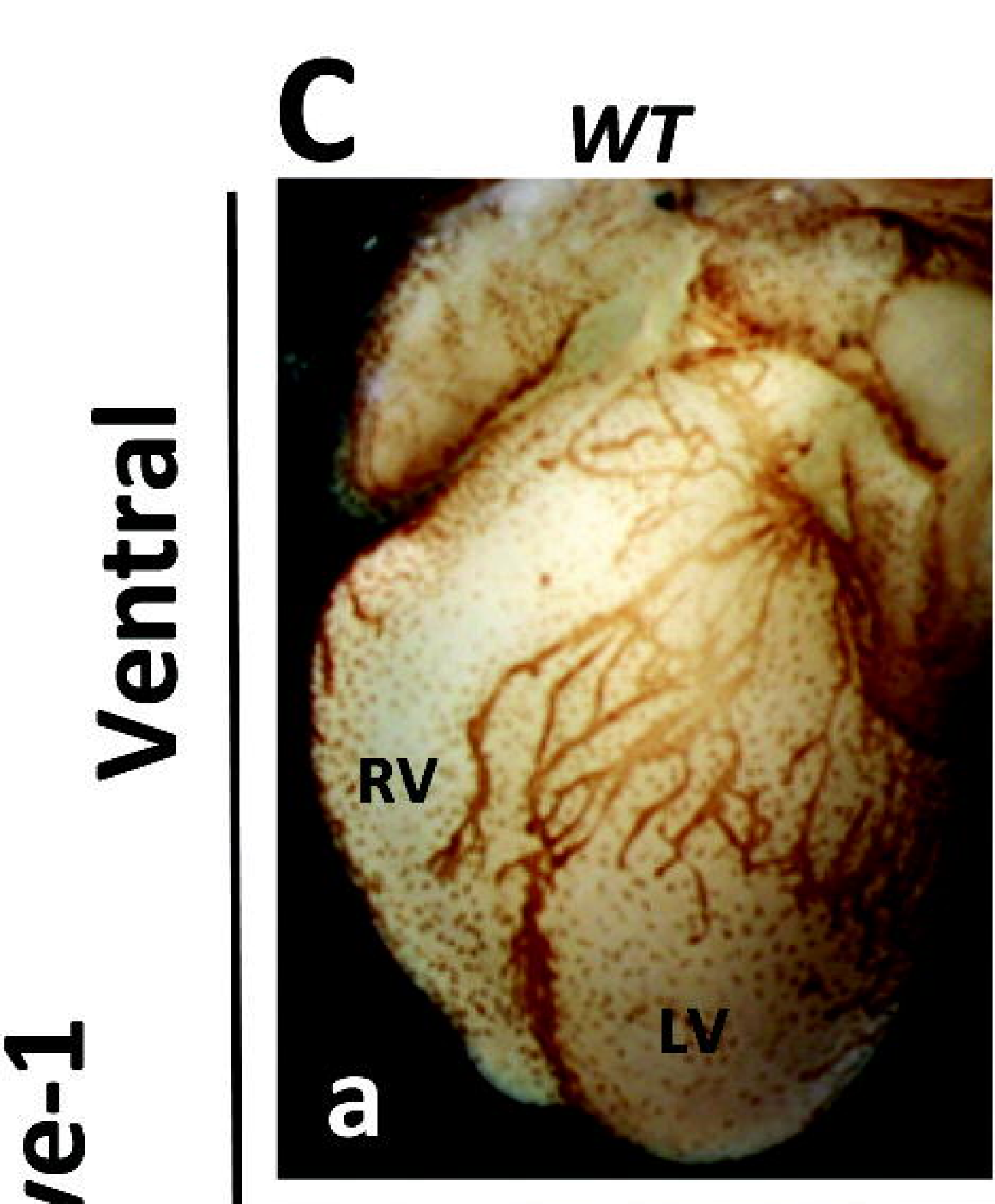

ত্তু
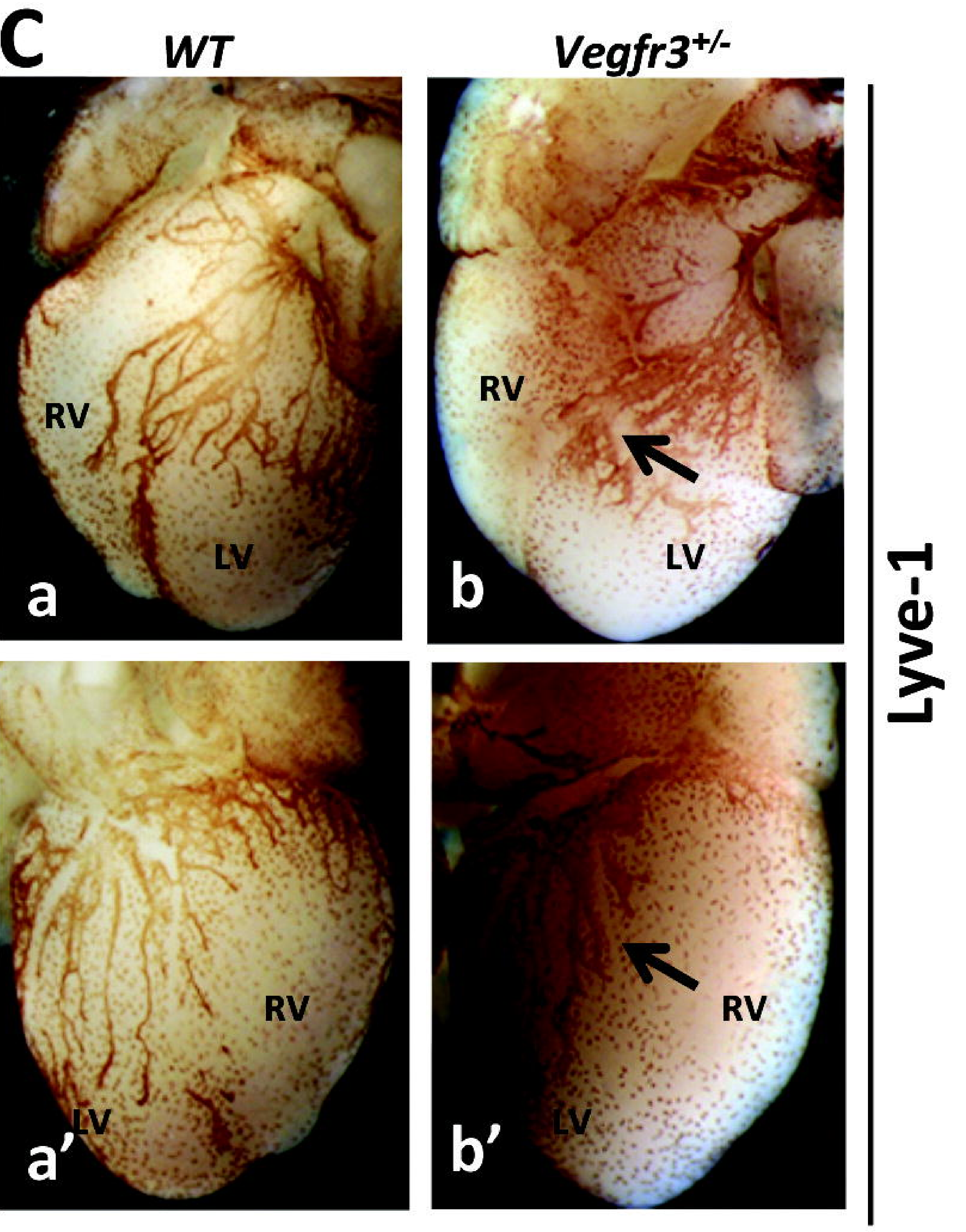

E 18.5
D
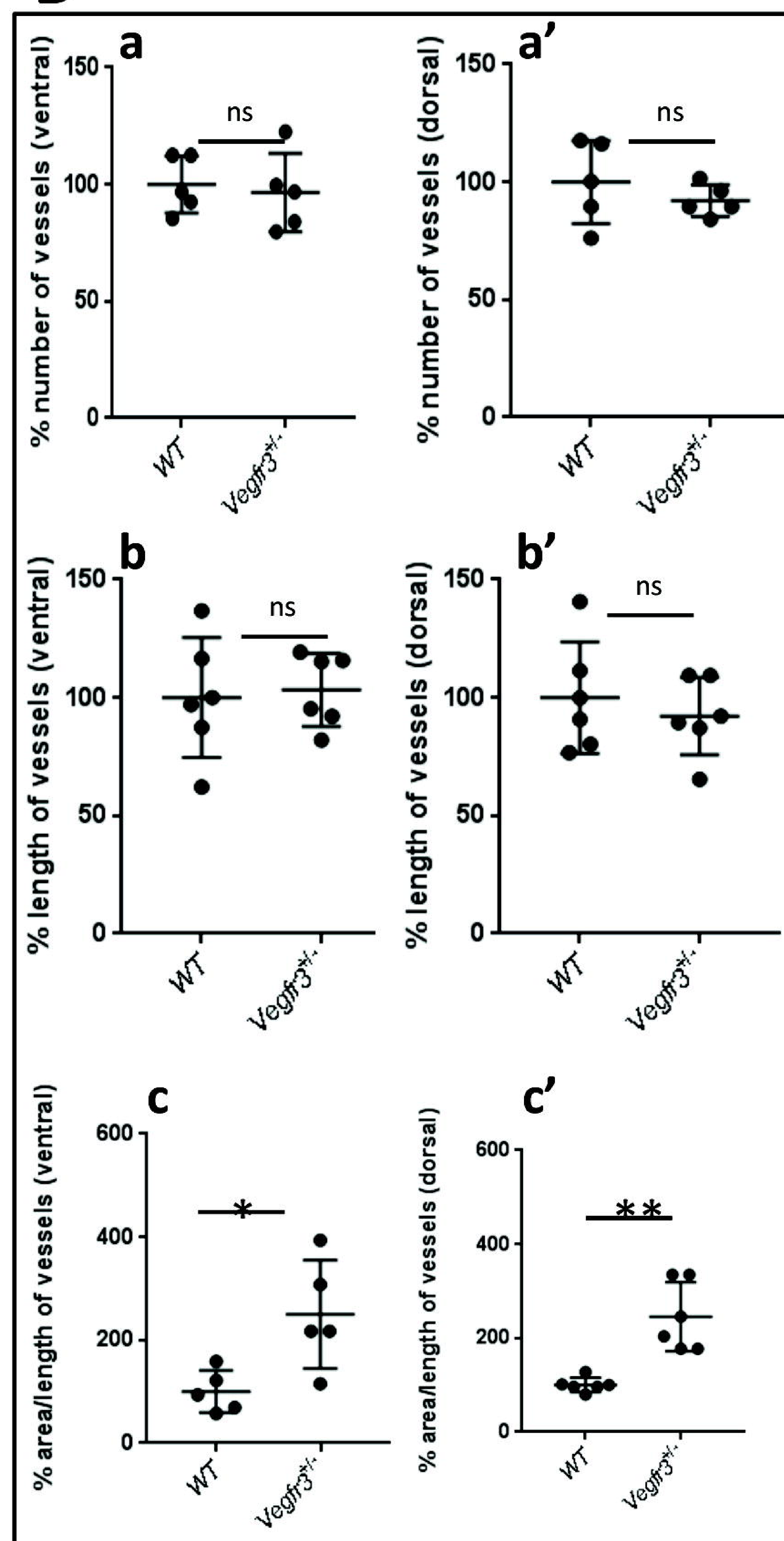


\section{A}
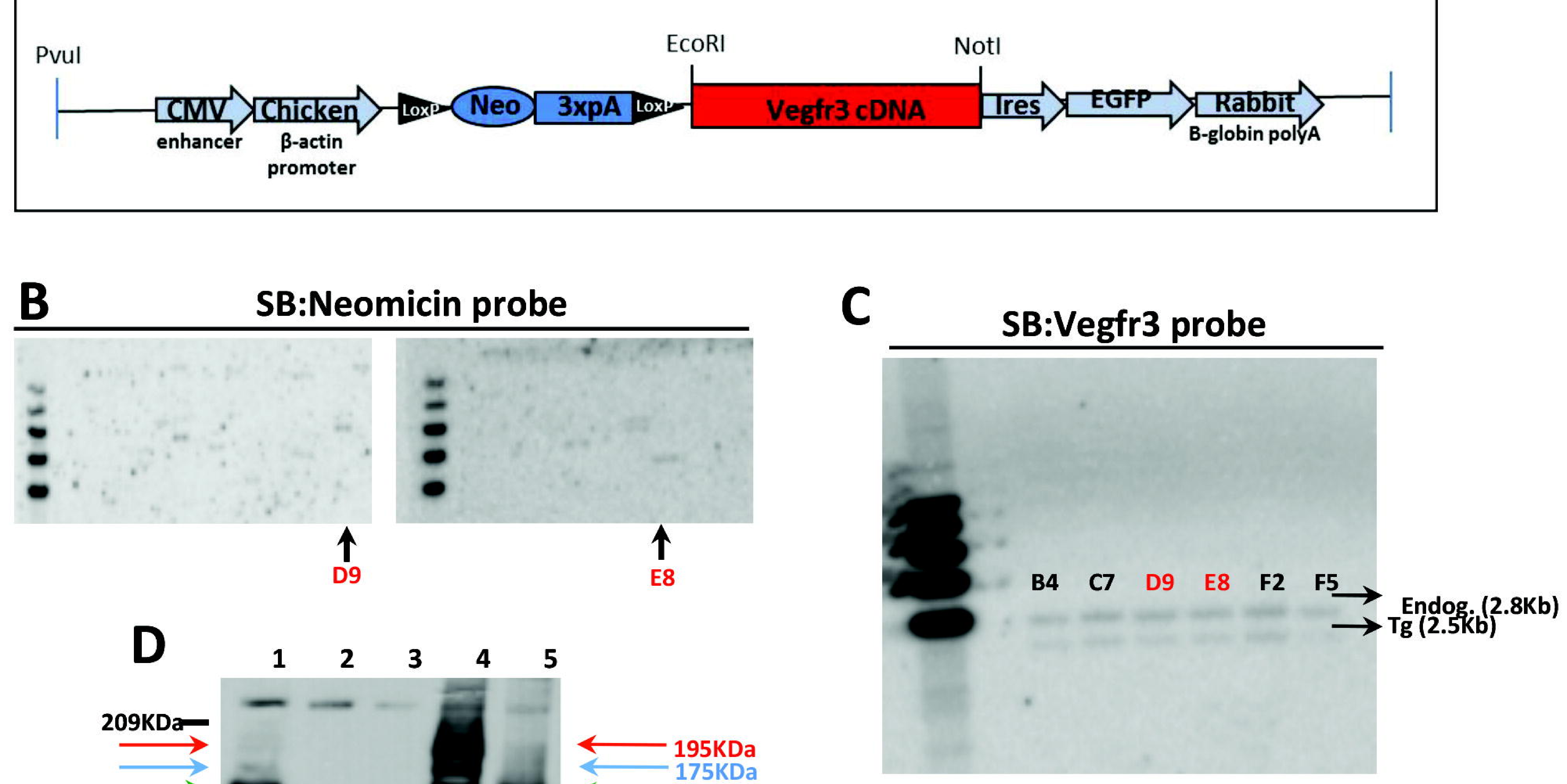

E
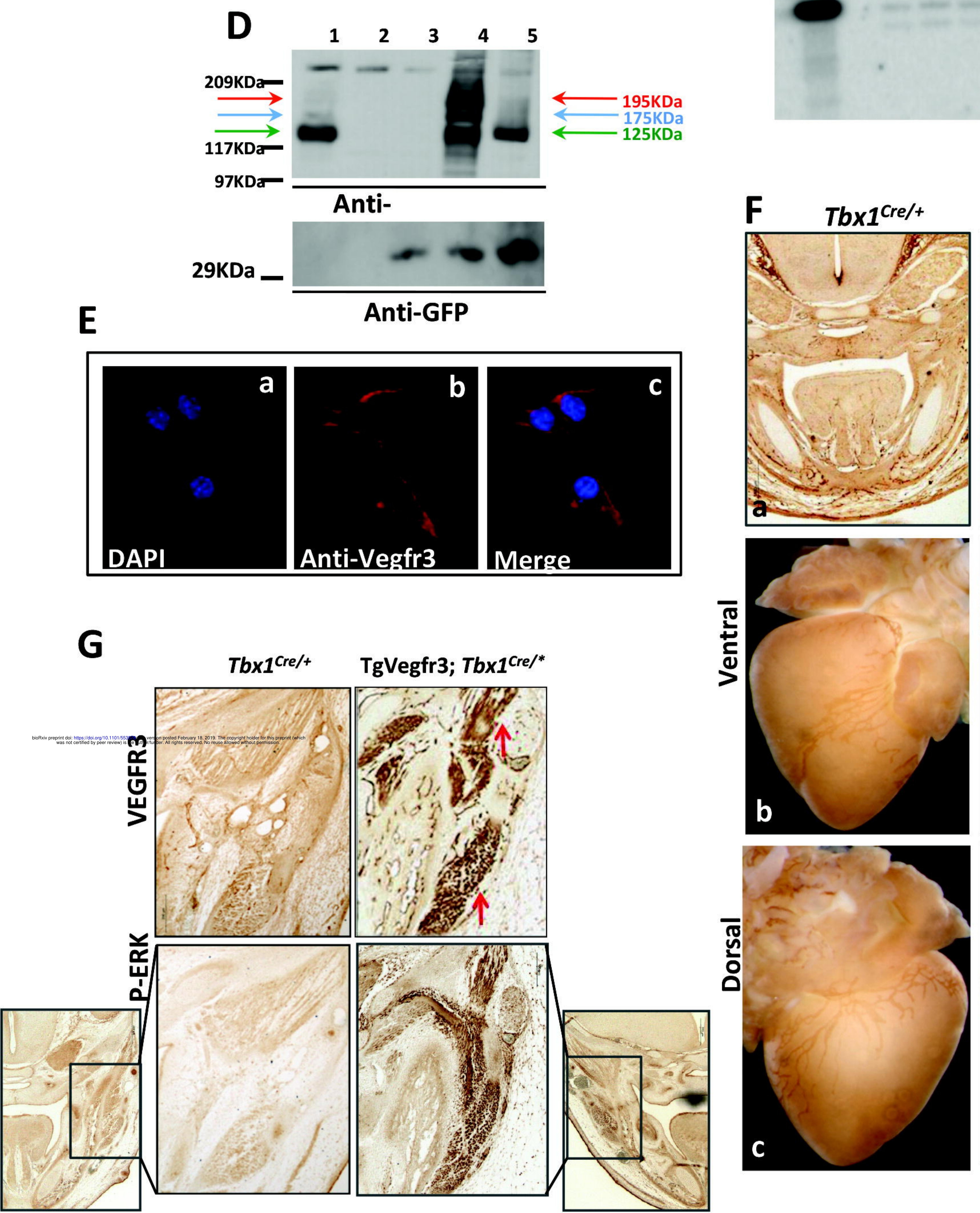

TgVegfr3; Tbx1 ${ }^{\text {Cre/* }}$
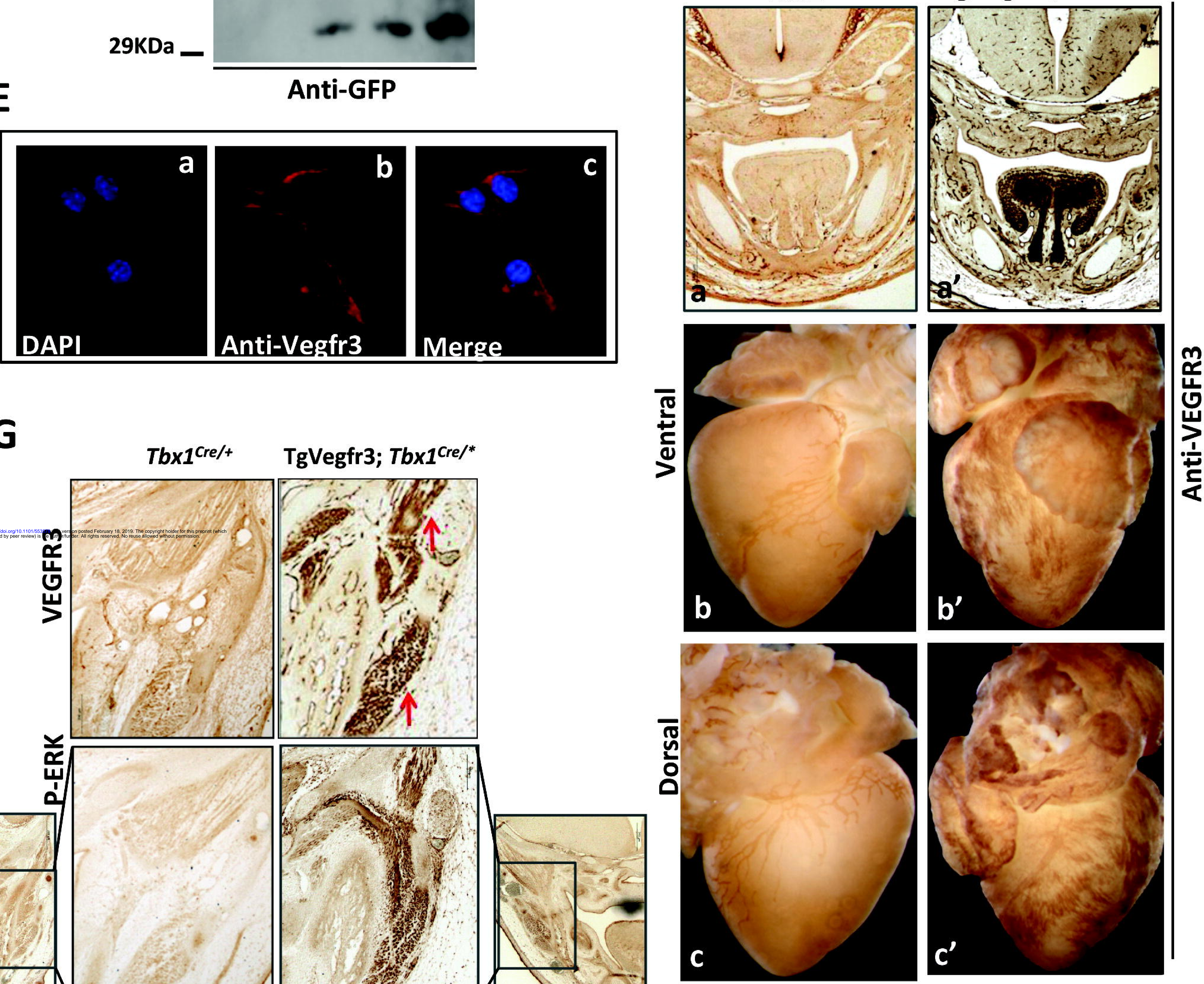


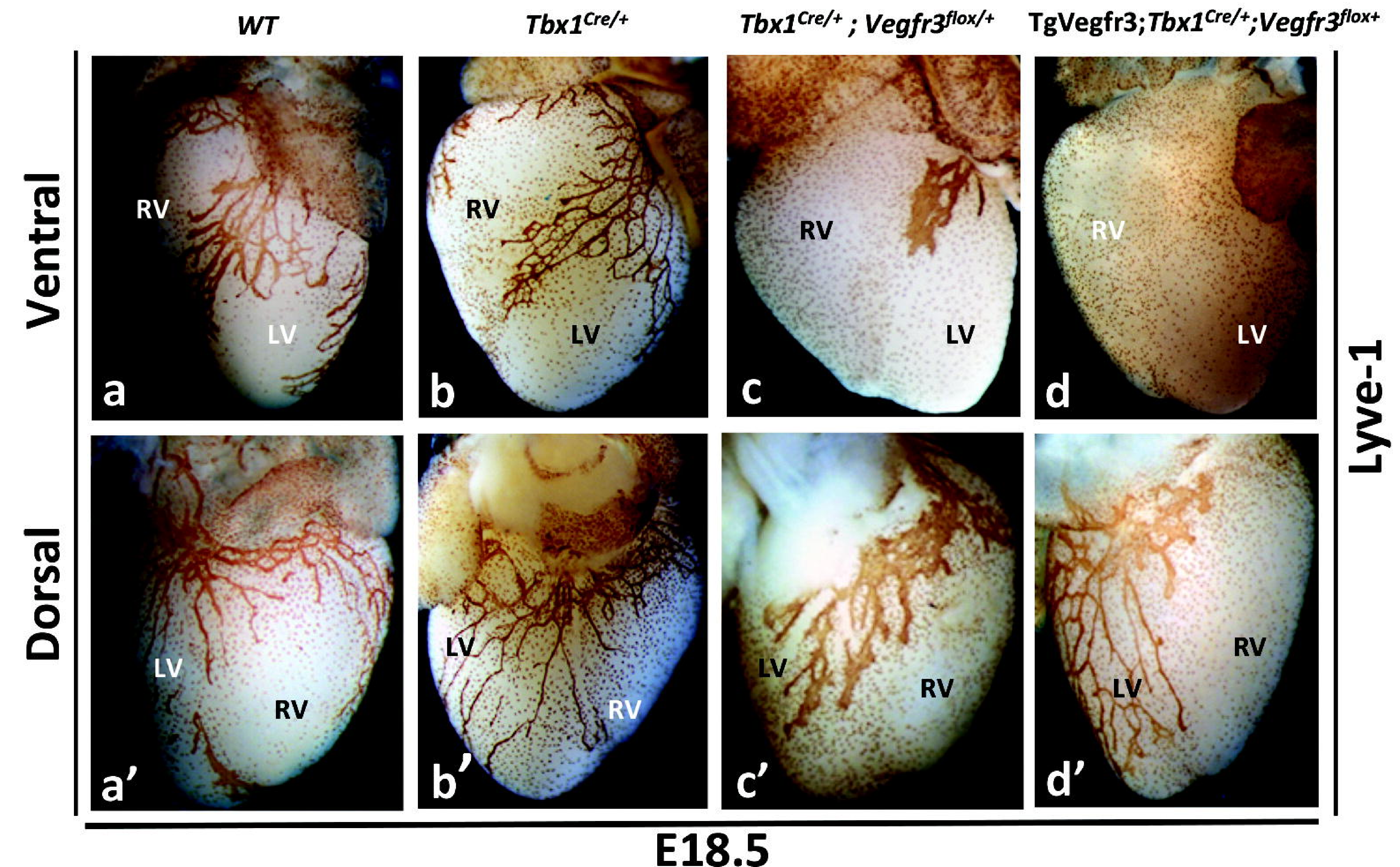

B
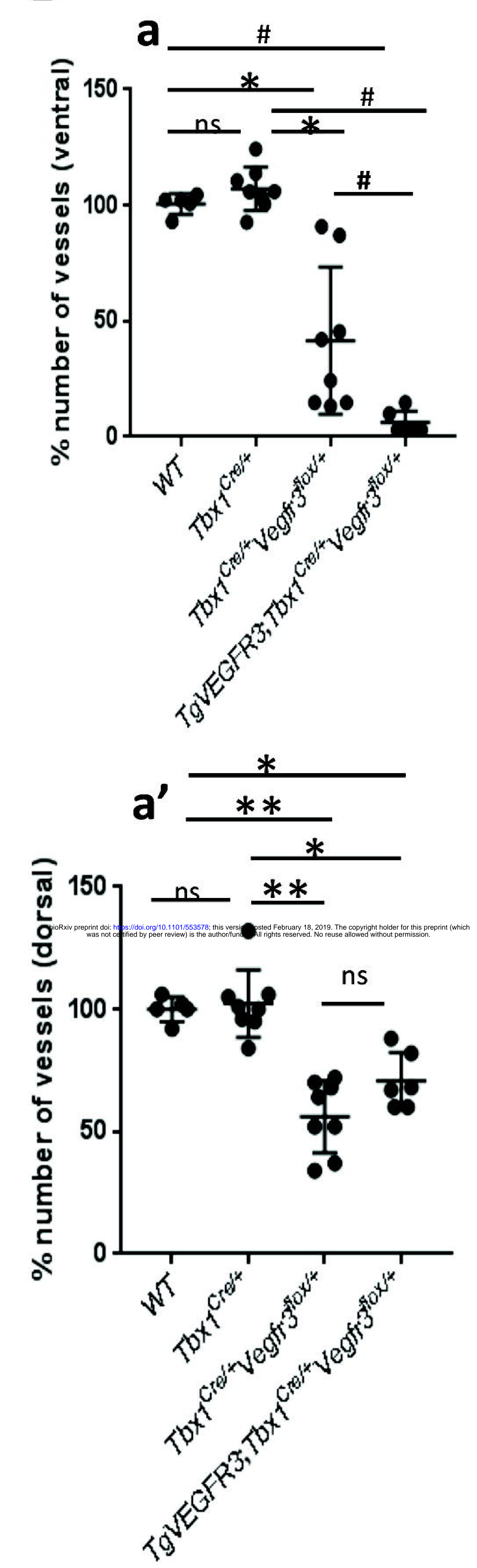
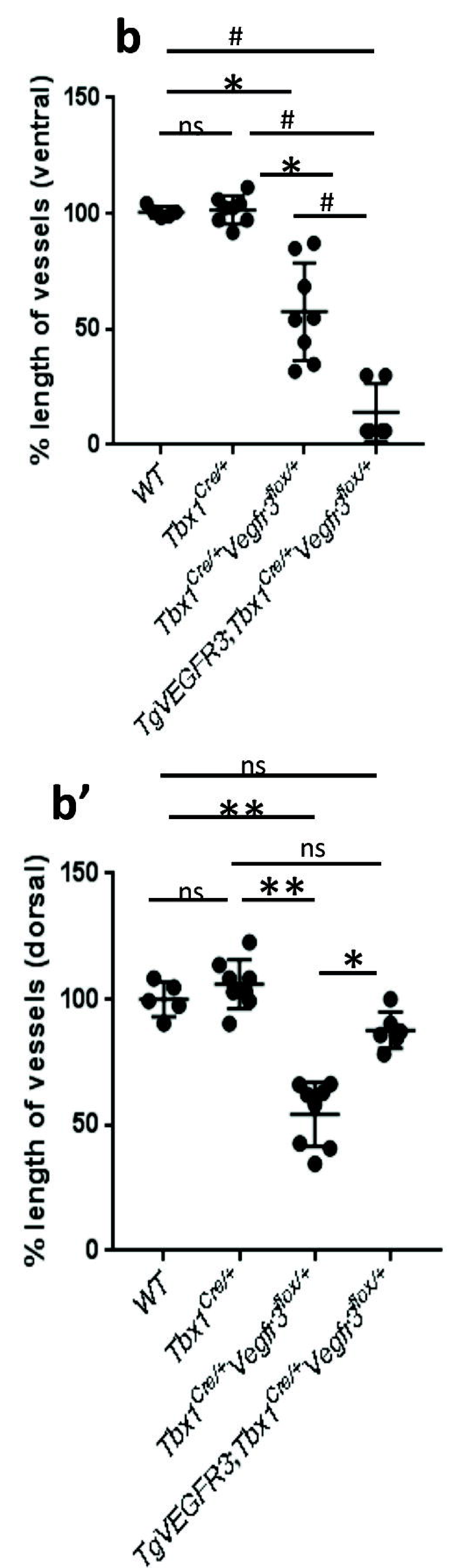
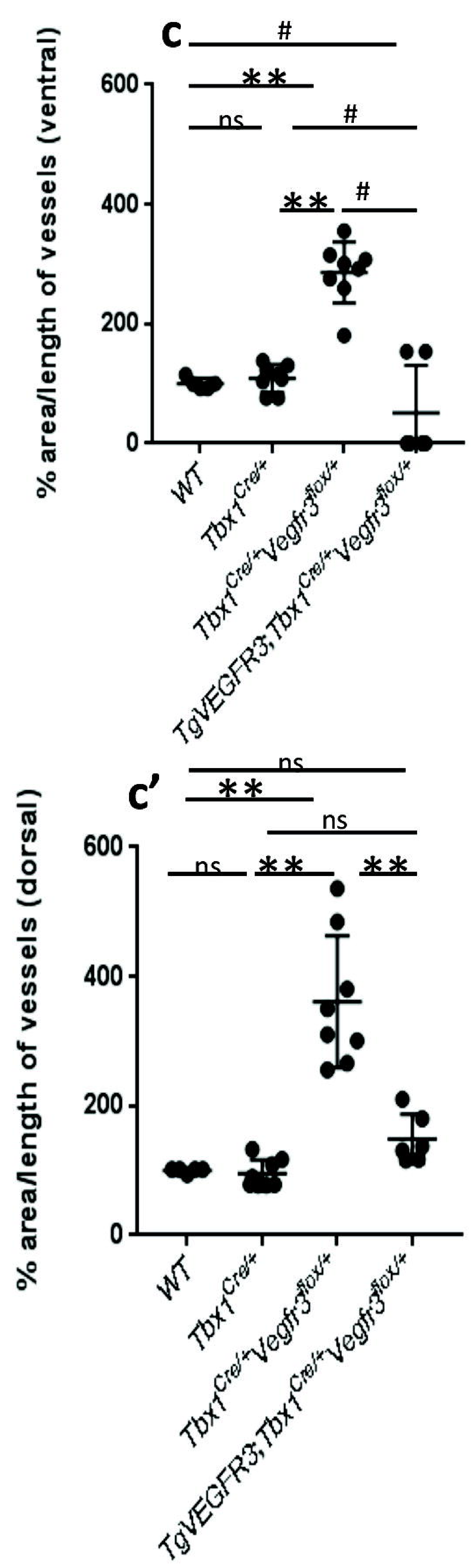